\title{
Enantioselective Iridium-Catalyzed Allylic Amination of Ammonia and Ammonia Surrogates
}

\section{Supporting Information}

Mark J. Pouy, Andreas Leitner, Daniel J. Weix, Satoshi Ueno and John F. Hartwig*

Department of Chemistry, University of Illinois, 600 South Mathews Avenue, Urbana, Illinois, 61801 and Department of Chemistry, and Yale University, P. O. Box 208107, New Haven, Connecticut, 06520

jhartwig@uiuc.edu 
General: All manipulations were carried out under an inert atmosphere using a nitrogen-filled glovebox or standard Schlenk techniques. All glassware was oven dried immediately prior to use. Tetrahydrofuran was degassed by purging with argon for 45 min and dried with a solvent purification system containing a $1 \mathrm{~m}$ column containing activated alumina. Propylamine was stirred over $\mathrm{CaH}_{2}$ for $24 \mathrm{~h}$ and distilled under nitrogen at reduced pressure. All other reagents were obtained from commercial sources and used without further purification. $\left[\operatorname{Ir}(\mathrm{COD}) \mathrm{Cl}_{2}{ }_{2}{ }^{1}\right.$ and phosphoramidite ligands ${ }^{2}$ were prepared according to published procedures.

All allylic carbonates were synthesized from the reaction of the corresponding allylic alcohol with methyl chloroformate in the presence of pyridine. (E)-4-methoxycinnamyl alcohol and (E)-3-(2-furanyl)-2-propen-1-ol were prepared by reduction of the corresponding aldehyde with $\mathrm{NaBH}_{4}$. (E)-3-cyclohexylprop-2-en-1-ol was synthesized by the Roush-Masamune modification of the Horner-Wadsworth-Emmons reaction. ${ }^{3}$ (E)-3-p-tolylprop-2-en-1-ol, (E)-3-(4-chlorophenyl)prop-2-en-1-ol, $(E)-3-(4-$ bromophenyl)prop-2-en-1-ol, and (E)-3-(2-fluorophenyl)prop-2-en-1-ol were prepared from the commercially available carboxylic acid by forming the mixed anhydride with ethyl chloroformate in the presence of pyridine followed by reduction with $\mathrm{NaBH}_{4}$.

Elemental analyses were performed by the University of Illinois at UrbanaChampaign Microanalysis laboratory and by Robertson Microlit Laboratories, Inc. (Madison, NJ). HRMS analyses were performed by the University of Illinois at UrbanaChampaign Mass Spectrometry laboratory. NMR spectra were obtained on $500 \mathrm{MHz}$ Varian Unity or Innova instruments (University of Illinois VOICE NMR facility) and worked up using the MestReNova software program. Chemical shifts are recorded relative to residual solvent peak $\left(\mathrm{CDCl}_{3}=7.26 \mathrm{ppm}\right.$ for ${ }^{1} \mathrm{H}, 77.0 \mathrm{ppm}$ for $\left.{ }^{13} \mathrm{C}\right)$ or to external standard $\left(\mathrm{CFCl}_{3}=0 \mathrm{ppm}\right.$ for $\left.{ }^{19} \mathrm{~F}\right)$. HPLC analyses were carried out on a Waters chromatography system (1525 binary pump, 717+ autosampler, 2487 dual wavelength detector).

Flash chromatography was conducted on Silicycle Siala-P Silica gel using ethyl acetate in hexanes in proportions indicated below. Products were visualized on TLC via UV or via $\mathrm{KMnO}_{4}$ stain as appropriate. 


\section{General Procedures for Enantioselective Allylic Amination.}

Catalyst activation with propylamine. For use in $0.5 \mathrm{mmol}$ amination reactions, $[\operatorname{lr}(\mathrm{COD}) \mathrm{Cl}]_{2}(0.0050 \mathrm{mmol})$ and the phosphoramidite ligand $(0.010 \mathrm{mmol})$ were dissolved in $0.1 \mathrm{ml}$ of THF and $0.2 \mathrm{ml}$ of propylamine. The mixture was heated at $50^{\circ} \mathrm{C}$ for $30 \mathrm{~min}$. After this time, all volatile materials were evaporated under vacuum, leaving the activated catalyst as a yellow solid. For reactions of ammonia, the remaining amine hydrochloride was removed by precipitation as described previously. ${ }^{4}$

Diallylation of Ammonia with Cinnamyl Carbonate. In a drybox, the isolated cyclometalated catalyst generated as described above (13.9 $\mathrm{mg}, 0.0101 \mathrm{mmol})$ and $[\operatorname{lr}(\mathrm{COD}) \mathrm{Cl}]_{2}(3.4 \mathrm{mg}, 0.0051 \mathrm{mmol})$ were added to a vial, which was sealed with a PTFE septum. Outside the glovebox, ammonia in ethanol $(2 \mathrm{M}, 0.5 \mathrm{~mL})$ was added to the vial, followed by methyl cinnamyl carbonate $(94.6 \mathrm{mg}, 0.492 \mathrm{mmol})$ in THF $(0.5 \mathrm{~mL})$. The reaction was stirred at room temperature for $8 \mathrm{~h}$ and monitored by GC. Upon completion, the volatile materials were evaporated under vacuum, and the crude reaction mixture was purified by flash column chromatography on silica gel (9\% ethyl acetate in hexanes) to give the title compound (56.9 mg, $93 \%$ ). ${ }^{1} \mathrm{H}$ NMR analysis indicates a 94:6 ratio of diasteriomers. HPLC analysis indicated that the enantiomeric excess of the product was 99\% [Diacel CHIRALCEL AD-H $(0.46 \mathrm{~cm} \times 25 \mathrm{~cm})$ hexanes $/ 2$-propanol $=99 / 1$; flow rate $=1.0 \mathrm{~mL} / \mathrm{min}$; detection wavelength $=220 \mathrm{~nm}] .{ }^{1} \mathrm{H}$ NMR $\delta$ 7.39-7.29 (m, 8H), 7.28-7.23 (m, 2H), 5.90 (ddd, $J=6.9,10.2,17.1 \mathrm{~Hz} 2 \mathrm{H}$ ), 5.18 $(\mathrm{d}, J=17.2 \mathrm{~Hz}, 2 \mathrm{H}), 5.11(\mathrm{~d} J=10.2 \mathrm{~Hz}, 2 \mathrm{H}), 4.17(\mathrm{~d}, J=6.9 \mathrm{~Hz}, 2 \mathrm{H}), 1.66(\mathrm{~s}, 1 \mathrm{H}) .{ }^{13} \mathrm{C}$ NMR $\delta 142.7,140.9,128.5,127.4,127.1,115.0,62.2$.

Catalytic Allylic Amination Reaction with Ammonia Surrogates. In a drybox, potassium trifluoroacetamide or the lithium salt of di-tert-butyliminodicarboxylate (0.6 $\mathrm{mmol}$ ) and hexamethylbenzene as an NMR standard were added to a vial containing the activated catalyst prepared as described above. The carbonate was dissolved in THF and added to the reaction vial, using the remaining THF ( $0.5 \mathrm{ml}$ total) to wash any residual carbonate into the reaction vial. The vial was sealed with a PTFE septum and removed from the drybox. The reaction progress was monitored by GC. After 
completion, the reactions were diluted with hexanes $(1 \mathrm{~mL})$. The reactions of potassium trifluoroacetamide were quenched with deionized water $(150 \mu \mathrm{L})$. A portion of the reaction mixture was removed for ${ }^{1} \mathrm{H}-\mathrm{NMR}$ analysis to determine the regioselectivity. The solvent was evaporated from the reactions of $\mathrm{LiN}(\mathrm{Boc})_{2}$ before column chromatography. Silica was added to the crude reactions of potassium trifluoroacetamide, and the volatile materials were removed under vacuum. The crude reaction mixtures were purified by flash column chromatography on silica gel (hexanes/ethyl acetate unless otherwise specified).

2,2,2-trifluoro-N-(1-phenylallyl)acetamide (Table 2, entry 1). The general procedure was followed with cinnamy methyl carbonate $(96.2 \mathrm{mg}, 0.501 \mathrm{mmol})$ and K-TFAc (90.8

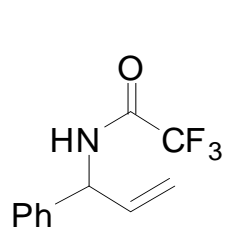

$\mathrm{mg}, 0.601 \mathrm{mmol})$. The reaction was conducted at room temperature for $8 \mathrm{~h}$. The mixture was purified by flash column chromatography on silica gel ( $9 \%$ ethyl acetate in hexanes) to give the title compound $(78.3 \mathrm{mg}$, $68 \%)$. HPLC analysis indicated that the enantiomeric excess of the product was 94\% [Diacel CHIRALCEL AD-H $(0.46 \mathrm{~cm} \times 25 \mathrm{~cm})$ hexanes/2-propanol = 99/1; flow rate $=1.0 \mathrm{~mL} / \mathrm{min}$; detection wavelength $=220 \mathrm{~nm}] .{ }^{1} \mathrm{H} \mathrm{NMR}\left(\mathrm{CDCl}_{3}\right) \delta 7.26-$ $2.44(\mathrm{~m}, 5 \mathrm{H}), 6.72$ (bs, $1 \mathrm{H}), 6.03$ (ddd, $J=5.5,10.4,15.9 \mathrm{~Hz}, 1 \mathrm{H}), 5.56$ (bt, $J=6.8 \mathrm{~Hz}$, $1 \mathrm{H}), 5.35(\mathrm{~d}, J=104 \mathrm{~Hz}, 1 \mathrm{H}), 5.27(\mathrm{~d}, J=17.2 \mathrm{~Hz}, 1 \mathrm{H}) .{ }^{13} \mathrm{C} \mathrm{NMR}\left(\mathrm{CDCl}_{3}\right) \delta 156.3(\mathrm{q}, J$ $=38 \mathrm{~Hz}$ ), 138.4, 135.2, 129.0, 128.4, 127.2, 117.3, 115.8 (q, $J=288 \mathrm{~Hz}$ ), 55.8. ${ }^{19} \mathrm{~F}$ NMR $\left(\mathrm{CDCl}_{3}\right) \delta$-75.9. Anal. Calcd. for $\left[\mathrm{C}_{11} \mathrm{H}_{10} \mathrm{~F}_{3} \mathrm{NO}\right]$ : C, 57.64; $\mathrm{H}, 4.40 ; \mathrm{N}, 6.11$ Found: C, 57.54; H, 4.33; N, 6.03 .

\section{2,2,2-trifluoro-N-(1-p-tolylallyl)acetamide (Table 2, entry 2). The} (1) general procedure was followed with $p$-tolyl cinnamyl methyl carbonate (103 mg, $0.499 \mathrm{mmol})$ and K-TFAc (90.1 mg, 0.596 $\mathrm{mmol})$. The reaction was conducted at room temperature for $5 \mathrm{~h}$. The mixture was purified by flash column chromatography on silica gel ( $9 \%$ ethyl acetate in hexanes) to give the title compound $(89.5 \mathrm{mg}, 74 \%)$. HPLC analysis indicated that the enantiomeric excess of the product was $96 \%$ [Diacel 
CHIRALCEL AD-H $(0.46 \mathrm{~cm} \times 25 \mathrm{~cm})$ hexanes/2-propanol $=99 / 1$; flow rate $=1.0$ $\mathrm{mL} / \mathrm{min}$; detection wavelength $=210 \mathrm{~nm}] .{ }^{1} \mathrm{H} \mathrm{NMR}\left(\mathrm{CDCl}_{3}\right) \delta 7.20(\mathrm{~s}, 4 \mathrm{H}), 6.55(\mathrm{bs}, 1 \mathrm{H})$, 6.02 (ddd, $J=5.38,10.4,17.1 \mathrm{~Hz}, 1 \mathrm{H}$ ), 5.50 (bt, $J=13.6 \mathrm{~Hz}, 1 \mathrm{H}$ ), 5.34 (ddd, $J=0.63$, 1.58, $10.4 \mathrm{~Hz}, 1 \mathrm{H}$ ), 5.26 (ddd, $J=0.63,1.68,17.2 \mathrm{~Hz}, 1 \mathrm{H}), 2.36(\mathrm{~s}, 3 \mathrm{H}) .{ }^{13} \mathrm{C} N M R$ $\left(\mathrm{CDCl}_{3}\right) \delta 156.2(\mathrm{q}, J=37.4 \mathrm{~Hz}), 138.4,135.4,135.3,129.7,127.1,117.0,115.8(\mathrm{q}, J=$ 285.6 Hz), 55.5, 21.1. ${ }^{19} \mathrm{~F} \mathrm{NMR}\left(\mathrm{CDCl}_{3}\right) \delta$-75.9. Anal. Calcd. for $\left[\mathrm{C}_{12} \mathrm{H}_{12} \mathrm{~F}_{3} \mathrm{NO}\right]: \mathrm{C}$, 59.26; H, 4.97; N, 5.76 Found: C, 59.21; H, 4.87; N, 5.74.

2,2,2-trifluoro-N-(1-(4-methoxyphenyl)allyl)acetamide (Table 2, entry 3).The general procedure was followed with $p$-methoxy cinnamyl methyl carbonate $(112.1 \mathrm{mg}, 0.504$ $\mathrm{mmol})$ and $\mathrm{K}-\mathrm{TFAc}(89.9 \mathrm{mg}, 0.595 \mathrm{mmol})$. The reaction was
$\begin{aligned} & \text { purified by flash column chromatography on silica gel (13\% ethyl } \\ & \text { acetate in hexanes) to give the title compound (123.0 } \mathrm{mg}, 94 \%) \text {. }\end{aligned}$ HPLC analysis indicated that the enantiomeric excess of the product was $98 \%$ [Diacel CHIRALCEL AD-H $(0.46 \mathrm{~cm} \times 25 \mathrm{~cm})$ hexanes/2-propanol $=98 / 2$; flow rate $=1.0$ $\mathrm{mL} / \mathrm{min}$; detection wavelength $=220 \mathrm{~nm}$. ${ }^{1} \mathrm{H}$ NMR $\left(\mathrm{CDCl}_{3}\right) \delta 7.22(\mathrm{~d}, J=8.65 \mathrm{~Hz}, 2 \mathrm{H})$, 6.90 (d, $J=8.65 \mathrm{~Hz}, 2 \mathrm{H}$ ), 6.56 (bs, 1H), 6.00 (ddd, $J=5.35,10.4,17.2 \mathrm{~Hz}, 1 \mathrm{H}$ ), 5.58 (bt, $J=6.6 \mathrm{~Hz}, 1 \mathrm{H}$ ), $5.33(\mathrm{~d}, J=10.3 \mathrm{~Hz}, 1 \mathrm{H}), 5.25(\mathrm{~d}, J=17.2 \mathrm{~Hz}, 1 \mathrm{H}), 3.81(\mathrm{~s}, 3 \mathrm{H})$. ${ }^{13} \mathrm{C}$ NMR $\left(\mathrm{CDCl}_{3}\right) \delta 159.8,156.4(\mathrm{q}, J=37.3 \mathrm{~Hz}), 135.6,130.6,128.8,117.0,116.0$ (q, $J$ $=288.1 \mathrm{~Hz})$, 114.6, 55.49, 55.40. ${ }^{19} \mathrm{~F} \mathrm{NMR}\left(\mathrm{CDCl}_{3}\right) \delta-76.49$. Anal. Calcd. for $\left[\mathrm{C}_{12} \mathrm{H}_{12} \mathrm{~F}_{3} \mathrm{NO}_{2}\right]$ : C, 55.60; $\mathrm{H}, 4.67 ; \mathrm{N}, 5.40$ Found: $\mathrm{C}, 55.82 ; \mathrm{H}, 4.47 ; \mathrm{N}, 5.19$.

N-(1-(4-chlorophenyl)allyl)-2,2,2-trifluoroacetamide (Table 2, entry 4). The general procedure was followed with $p$-chloro cinnamy methyl carbonate $(112.1 \mathrm{mg}, 0.495$ (l) $\mathrm{mmol}$ ) and K-TFAc $(90.2 \mathrm{mg}, 0.597 \mathrm{mmol})$. The reaction was conducted at room temperature for $12 \mathrm{~h}$. The mixture was purified by flash column chromatography on silica gel ( $9 \%$ ethyl acetate in hexanes) to give the title compound ( $85 \mathrm{mg}, 65 \%)$. HPLC analysis indicated that the enantiomeric excess of the product was $94 \%$ [Diacel CHIRALCEL AD- 
$\mathrm{H}(0.46 \mathrm{~cm} \times 25 \mathrm{~cm})$ hexanes $/ 2$-propanol $=97 / 3$; flow rate $=1.0 \mathrm{~mL} / \mathrm{min}$; detection wavelength $=220 \mathrm{~nm}] .{ }^{1} \mathrm{H} \mathrm{NMR}\left(\mathrm{CDCl}_{3}\right) \delta 7.35(\mathrm{~d}, J=8.5 \mathrm{~Hz}, 2 \mathrm{H}), 7.23(\mathrm{~d}, J=8.4 \mathrm{~Hz}$, 1H), 6.70 (bs, $1 \mathrm{H}$ ), 5.99 (ddd, $J=5.6,10.4,16.0,1 \mathrm{H}$ ), 5.59 (m, 1H), 5.36 (dd, $J=1.5$, $10.4 \mathrm{~Hz}, 1 \mathrm{H}), 5.26(\mathrm{dd}, J=1.6,17.1 \mathrm{~Hz}, 1 \mathrm{H}) .{ }^{13} \mathrm{C} \mathrm{NMR}\left(\mathrm{CDCl}_{3}\right) \delta 156.4(\mathrm{q}, J=37 \mathrm{~Hz})$, 136.9, 134.7, 134.3, 129.2, 128.6, 118.0, 115.7, 55.1. ${ }^{19} \mathrm{~F} \mathrm{NMR}\left(\mathrm{CDCl}_{3}\right) \delta$-75.9. Anal. Calcd. for $\left[\mathrm{C}_{11} \mathrm{H}_{9} \mathrm{ClF}_{3} \mathrm{NO}\right]: \mathrm{C}, 50.11 ; \mathrm{H}, 3.44 ; \mathrm{N}, 5.31$ Found: $\mathrm{C}, 50.36 ; \mathrm{H}, 3.36 ; \mathrm{N}, 5.10$.

N-(1-(4-bromophenyl)allyl)-2,2,2-trifluoroacetamide (Table 2, entry 5). The general procedure was followed with $p$-bromo cinnamy methyl carbonate (135.4 mg, 0.499 $\mathrm{mmol}$ ) and K-TFAc (91.3 $\mathrm{mg}, 0.604 \mathrm{mmol})$. The reaction was (N) conducted at room temperature for $18 \mathrm{~h}$. The mixture was purified by flash column chromatography on silica gel ( $9 \%$ ethyl acetate in hexanes) to give the title compound ( $89.5 \mathrm{mg}, 58 \%$ yield). HPLC analysis indicated that the enantiomeric excess of the product was 92\% [[Diacel CHIRALCEL AD-H $(0.46 \mathrm{~cm} \times 25 \mathrm{~cm})$ hexanes/2-propanol = 98/2; flow rate $=1.0 \mathrm{~mL} / \mathrm{min}$; detection wavelength $=220 \mathrm{~nm}] .{ }^{1} \mathrm{H} \mathrm{NMR}\left(\mathrm{CDCl}_{3}\right) \delta 7.51(\mathrm{~d}, J=8.5 \mathrm{~Hz}$, 2H), 7.17 (d, $J=8.3 \mathrm{~Hz}, 2 \mathrm{H}$ ), 6.58 (bs, $1 \mathrm{H}$ ), 5.99 (ddd, $J=5.5,10.4,17.1 \mathrm{~Hz}, 1 \mathrm{H}$ ), 5.58 (bt, $J=6.8 \mathrm{~Hz}, 1 \mathrm{H}$ ), 5.37 (dd, $J=1.2,10.4 \mathrm{~Hz}, 1 \mathrm{H}$ ), 5.26 (dd, $J=1.3,17.1 \mathrm{~Hz}, 1 \mathrm{H}$ ). ${ }^{13} \mathrm{C}$ $\operatorname{NMR}\left(\mathrm{CDCl}_{3}\right) \delta 156.3$ (q, $\left.J=32 \mathrm{~Hz}\right), 137.4,134.7,132.2,128.9,122.5,118.0,115.7$ (q, $J=288 \mathrm{~Hz})$, 55.2. ${ }^{19} \mathrm{~F} \mathrm{NMR}\left(\mathrm{CDCl}_{3}\right) \delta$-75.9. HRMS $\left[\mathrm{C}_{11} \mathrm{H}_{9} \mathrm{BrF}_{3} \mathrm{NO}\right] \mathrm{M}=306.9871$; found 306.9822 .

2,2,2-trifluoro-N-(1-(2-fluorophenyl)allyl)acetamide (Table 2, entry 6). The general

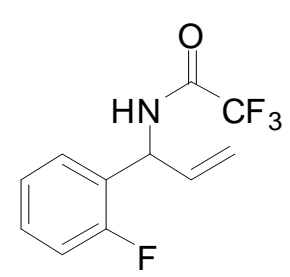
procedure was followed with o-fluoro cinnamy methyl carbonate (105.1 $\mathrm{mg}, 0.502 \mathrm{mmol})$ and K-TFAc $(90.8 \mathrm{mg}, 0.601 \mathrm{mmol})$. The reaction was conducted at room temperature for $18 \mathrm{~h}$. The mixture was purified by flash column chromatography on silica gel (9\% ethyl acetate in hexanes) to give the title compound $(60.0 \mathrm{mg}, 48 \%)$. HPLC analysis indicated that the enantiomeric excess of the product was $84 \%$ [Diacel CHIRALCEL AD-H $(0.46 \mathrm{~cm} \times 25$ $\mathrm{cm}$ ) hexanes $/ 2$-propanol $=99 / 1$; flow rate $=1.0 \mathrm{~mL} / \mathrm{min}$; detection wavelength $=210$ 
nm]. ${ }^{1} \mathrm{H}$ NMR $\left(\mathrm{CDCl}_{3}\right) \delta$ 7.38-7.32 $(\mathrm{m}, 1 \mathrm{H}), 7.29(\mathrm{dt}, J=1.7,7.6 \mathrm{~Hz}, 1 \mathrm{H}), 7.16(\mathrm{dt}, J=$ 1.2, $7.5 \mathrm{~Hz}, 1 \mathrm{H}$ ), 7.11 (ddd, $J=1.1,8.3,10.8 \mathrm{~Hz}, 1 \mathrm{H}$ ), 6.87 (bs, $1 \mathrm{H}$ ), 6.02 (dddd, $J=$ 0.76, 5.4, 10.3, $16.6 \mathrm{~Hz}, 1 \mathrm{H}$ ), 5.77 (bt, $J=7.0,1 \mathrm{H}$ ), 5.31 (dd, $J=1.3,10.3 \mathrm{~Hz}, 1 \mathrm{H}$ ), 5.21 (dd, $J=1.7,17.1,1 \mathrm{H}) .{ }^{13} \mathrm{C} \mathrm{NMR}\left(\mathrm{CDCl}_{3}\right) \delta 160.7$ (d, $\left.J=247 \mathrm{~Hz}\right), 160.7$ (q, $J=37 \mathrm{~Hz}$ ), 134.4, 130.3 (d, $J=8.5 \mathrm{~Hz}$ ), 129.2 (d, $J=4.2 \mathrm{~Hz}$ ), 125.4 (d, $J=13.1 \mathrm{~Hz}), 124.7$ (d, $J=$ $3.5 \mathrm{~Hz}), 117.6,116.2(\mathrm{~d}, J=21.3 \mathrm{~Hz}), 115.7(\mathrm{q}, J=288 \mathrm{~Hz}), 52.1 .{ }^{19} \mathrm{~F} \mathrm{NMR}\left(\mathrm{CDCl}_{3}\right) \delta-$ 76.1 (3F), -117.5 (1F). Anal. Calcd. for $\left[\mathrm{C}_{11} \mathrm{H}_{9} \mathrm{~F}_{4} \mathrm{NO}\right]: \mathrm{C}, 53.45 ; \mathrm{H}, 3.67 ; \mathrm{N}, 5.67$ Found: C, 53.46; H, 3.42; N, 5.58 .

$\mathrm{N}$-(dec-1-en-3-yl)-2,2,2-trifluoroacetamide (Table 2, entry 7). The general procedure was followed with $(E)$-dec-2-enyl methyl carbonate $(106 \mathrm{mg}, 0.495 \mathrm{mmol})$ and K-TFAc ${ }^{\mathrm{C}}$ $(90.7 \mathrm{mg}, 0.600 \mathrm{mmol})$. The reaction was conducted at room hexanes) to give the title compound $(75.9 \mathrm{mg}, 61 \%)$. HPLC analysis indicated that the enantiomeric excess of the product was 94\% [Diacel CHIRALCEL AS-H $(0.46 \mathrm{~cm} \times 25$ $\mathrm{cm}$ ) hexanes $/ 2$-propanol $=99.5 / 0.5 ;$ flow rate $=1.0 \mathrm{~mL} / \mathrm{min}$; detection wavelength $=210$ nm]. ${ }^{1} \mathrm{H}$ NMR $\left(500 \mathrm{MHz}, \mathrm{CDCl}_{3}\right) \delta 6.08(\mathrm{br} \mathrm{s}, 1 \mathrm{H}), 5.76$ (ddd, $J=17.2,10.5,6.1 \mathrm{~Hz}$, $1 \mathrm{H}$ ), 5.23-5.19 (m, 2H), 4.46 (quintet, $J=7.2 \mathrm{~Hz}, 1 \mathrm{H}), 1.67-1.53(\mathrm{~m}, 2 \mathrm{H}), 1.37-1.26(\mathrm{~m}$, $10 \mathrm{H}), 0.88$ (t, $J=7.0 \mathrm{~Hz}, 3 \mathrm{H}) .{ }^{13} \mathrm{C} \mathrm{NMR}\left(\mathrm{CDCl}_{3}\right) \delta 156.5(\mathrm{q}, J=37 \mathrm{~Hz}), 136.3,116.4$, $115.9(q, J=289 \mathrm{~Hz}), 52.4,34.3,31.7,29.14,29.05,25.5,22.6,14.0 .{ }^{19} \mathrm{~F} \operatorname{NMR}\left(\mathrm{CDCl}_{3}\right)$ $\delta$-76.2. Anal. Calcd. for $\left[\mathrm{C}_{12} \mathrm{H}_{20} \mathrm{~F}_{3} \mathrm{NO}\right]: \mathrm{C}, 59.26$; $\mathrm{H}, 4.97$; N, 5.76 Found: $\mathrm{C}, 59.21 ; \mathrm{H}$, $4.87 ; \mathrm{N}, 5.74$.

$\mathrm{N}$-(1-cyclohexylallyl)-2,2,2-trifluoroacetamide (Table 2, entry 8). The general procedure was followed with $(E)-3$-cyclohexylallyl methyl carbonate $(98.8 \mathrm{mg}, 0.4983$ $\mathrm{mmol})$ and $\mathrm{K}-\mathrm{TFAc}(90.3 \mathrm{mg}, 0.598 \mathrm{mmol})$ and using $4 \mathrm{~mol} \%$ iridium
and ligand instead of $2 \mathrm{~mol} \%$. The reaction was conducted at room
temperature for $18 \mathrm{~h}$. The mixture was purified by flash column 
chromatography on silica gel ( $9 \%$ ethyl acetate in hexanes) to give the title compound (93.6 $\mathrm{mg}, 80 \%)$. HPLC analysis indicated that the enantiomeric excess of the product was $97 \%$ [Diacel CHIRALCEL AD-H $(0.46 \mathrm{~cm} \times 25 \mathrm{~cm})$ hexanes/2-propanol = 99.5/0.5; flow rate $=1.0 \mathrm{~mL} / \mathrm{min}$; detection wavelength $=210 \mathrm{~nm}] .{ }^{1} \mathrm{H} \mathrm{NMR}\left(500 \mathrm{MHz}, \mathrm{CDCl}_{3}\right) \delta$ 6.31 (br s, 1H), 5.74 (ddd, J=17.0, 10.5, $6.7 \mathrm{~Hz}, 1 \mathrm{H}$ ), 5.21 (dt, J=10.5, 1.2 Hz, 1H), 5.18 (dt, J=17.1, 1.2 Hz, 1H), 4.30 (q, J=8.6 Hz, 1H), 1.78-1.65 (m, 5H), 1.51 (tdt, J=11.7, 6.6, 3.3 Hz, $1 \mathrm{H}), 1.27-1.08(\mathrm{~m}, 3 \mathrm{H}), 1.03-0.93(\mathrm{~m}, 2 \mathrm{H}) .{ }^{13} \mathrm{C} \mathrm{NMR}\left(\mathrm{CDCl}_{3}\right) \delta 156.6(\mathrm{q}$, $\mathrm{J}=36 \mathrm{~Hz}), 134.9,117.3,115.9$ (q, J=288 Hz), 57.2, 41.5, 29.2, 28.6, 26.1, $25.8(2 \mathrm{C}) .{ }^{19} \mathrm{~F}$ NMR $\left(\mathrm{CDCl}_{3}\right) \delta$-76.1. Anal. Calcd. for $\left[\mathrm{C}_{11} \mathrm{H}_{16} \mathrm{~F}_{3} \mathrm{NO}\right]$ : $\mathrm{C}, 59.26 ; \mathrm{H}, 4.97 ; \mathrm{N}, 5.76$ Found: C, $59.21 ; \mathrm{H}, 4.87 ; \mathrm{N}, 5.74$.

(Table 4, entry 1). The general procedure was followed with cinnamyl methyl carbonate $(192 \mathrm{mg}, 0.997 \mathrm{mmol})$ and $\mathrm{LiN}(\mathrm{Boc})_{2}(267.3 \mathrm{mg}, 1.198 \mathrm{mmol})$. The reaction

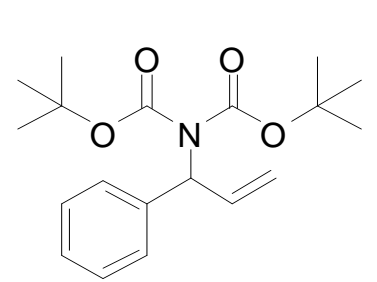
was conducted at room temperature for $2 \mathrm{~h}$. ${ }^{1} \mathrm{H}$ NMR analysis of the crude reaction mixture indicated the ratio of regioisomers $(b / l)$ to be $93 / 7$. The mixture was purified by flash column chromatography on silica gel (7\% ethyl acetate in hexanes) to give the title compound ( $274 \mathrm{mg}, 82 \%$ ). HPLC analysis indicated that the enantiomeric excess of the product was 93\% [Diacel CHIRALCEL AD-H $(0.46 \mathrm{~cm} \times 25 \mathrm{~cm})$ hexanes $/ 2$-propanol $=99.5 / 0.5$; flow rate $=1.0 \mathrm{~mL} / \mathrm{min}$; detection wavelength $=220 \mathrm{~nm}$ ] ${ }^{1} \mathrm{H}$ NMR $\left(500 \mathrm{MHz}, \mathrm{CDCl}_{3}\right) \delta$ 7.31-7.15 (m, 5H), 6.33 (ddd, J=18.0, 10.1, 8.0 Hz, $1 \mathrm{H}$ ), $5.86(\mathrm{~d}, J=8.0 \mathrm{~Hz}, 1 \mathrm{H}), 5.32-5.27(\mathrm{~m}, 2 \mathrm{H}), 1.31(\mathrm{~s}, 18 \mathrm{H}) .{ }^{13} \mathrm{C} \mathrm{NMR}\left(\mathrm{CDCl}_{3}\right) \delta 152.3$, $140.3,135.4,128.1,126.8,126.4,119.2,82.3,61.8,27.8$. Anal. Calcd. for $\left[\mathrm{C}_{19} \mathrm{H}_{27} \mathrm{NO}_{4}\right]$ : C, 59.26; H, 4.97; N, 5.76 Found: C, 59.21; H, 4.87; N, 5.74.

(Table 4, entry 2). The general procedure was followed with $p$-methoxy cinnamyl methyl carbonate $(111 \mathrm{mg}, 0.500 \mathrm{mmol})$ and $\mathrm{LiN}(\mathrm{Boc})_{2}(132 \mathrm{mg}, 0.594 \mathrm{mmol})$. The reaction was conducted at room temperature for $6 \mathrm{~h}$. ${ }^{1} \mathrm{H}$ NMR analysis of the crude reaction mixture indicated the ratio of regioisomers $(b / l)$ to be $93 / 7$. The mixture was purified by flash column chromatography on silica gel ( $11 \%$ ethyl acetate in hexanes) to 


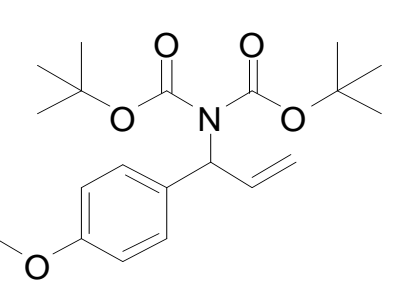

give the title compound (148 mg, 82\%). HPLC analysis after monodeprotection via treatment with trifluoroacetic acid indicated that the enantiomeric excess of the product was $94 \%$ [Diacel CHIRALCEL AD-H $(0.46 \mathrm{~cm} \times 25 \mathrm{~cm})$ hexanes/2propanol $=99 / 1 ;$ flow rate $=1.0 \mathrm{~mL} / \mathrm{min}$; detection wavelength $=220 \mathrm{~nm}] .{ }^{1} \mathrm{H}$ NMR $(500$ $\left.\mathrm{MHz}, \mathrm{CDCl}_{3}\right) \delta 7.24(\mathrm{~d}, J=8.3 \mathrm{~Hz}, 2 \mathrm{H}), 6.84(\mathrm{~d}, J=8.8 \mathrm{~Hz}, 2 \mathrm{H}), 6.36$ (ddd, $J=7.8$, 10.2, $17.3 \mathrm{~Hz}, 1 \mathrm{H}$ ), 5.84 (d, J = 7.7 Hz, 1H), 5.40-5.23 (m, 2H), $3.80(\mathrm{~s}, 3 \mathrm{H}), 1.374(\mathrm{~s}$, 18H). ${ }^{13} \mathrm{C}$ NMR $\left(\mathrm{CDCl}_{3}\right) \delta 158.5,152.5,135.7,132.2,127.9,118.7,113.4,82.2,61.3$, 55.2, 27.8. Anal. Calcd. for $\left[\mathrm{C}_{20} \mathrm{H}_{29} \mathrm{NO}_{5}\right]$ : C, 59.26; $\mathrm{H}, 4.97 ; \mathrm{N}, 5.76$ Found: C, 59.21; $\mathrm{H}$, $4.87 ; \mathrm{N}, 5.74$.

(Table 4, entry 3). The general procedure was followed with $p$-trifluoromethyl cinnamyl methyl carbonate $(129.4 \mathrm{mg}, 0.497 \mathrm{mmol})$ and $\mathrm{LiNBoc}_{2}(132 \mathrm{mg}, 0.592 \mathrm{mmol})$ and using

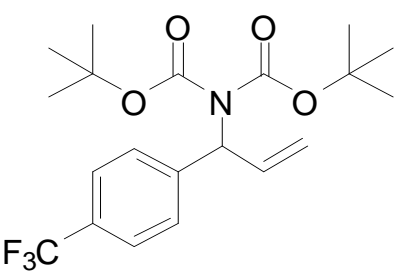
$3 \mathrm{~mol} \%$ iridium and ligand instead of $2 \mathrm{~mol} \%$. The reaction was conducted at room temperature for $4 \mathrm{~h} .{ }^{1} \mathrm{H}$ NMR analysis of the crude reaction mixture indicated the ratio of regioisomers (b/l) to be $86 / 14$. The mixture was purified by flash column chromatography on silica gel ( $7 \%$ ethyl acetate in hexanes) to give the title compound (152 mg, 76\%). HPLC analysis after monodeprotection via treatment with trifluoroacetic acid indicated that the enantiomeric excess of the product was 93\% [Diacel CHIRALCEL OD-H $(0.46 \mathrm{~cm} \times 25 \mathrm{~cm})$ hexanes $/ 2$-propanol $=99 / 1$; flow rate $=1.0 \mathrm{~mL} / \mathrm{min}$; detection wavelength $=210 \mathrm{~nm}] .{ }^{1} \mathrm{H} \mathrm{NMR}\left(\mathrm{CDCl}_{3}\right) \delta 7.58(\mathrm{~d}, J=8.2 \mathrm{~Hz}, 2 \mathrm{H}), 7.44(\mathrm{~d}, J=8.6 \mathrm{~Hz}$, 2H), 6.36, (ddd, $J=7.94,10.15,17.22,1 \mathrm{H}$ ), 5.93 (d, $J=7.8 \mathrm{~Hz}, 1 \mathrm{H}$ ), 5.42 (ddd, $J=$ $0.75,1.28,10.16 \mathrm{~Hz}, 1 \mathrm{H}), 5.37(\mathrm{td}, J=1.18,17.20,1 \mathrm{H}), 1.39(\mathrm{~s}, 18 \mathrm{H}) .{ }^{13} \mathrm{C}$ NMR $\left(\mathrm{CDCl}_{3}\right) \delta 152.2,144.5,134.6,129.2$ (q, $\left.32 \mathrm{~Hz}\right), 126,7,125.1$ (q, $\left.J=3.7 \mathrm{~Hz}\right), 124.1$ (q, $J$ $=272 \mathrm{~Hz}), 120.3,82.9,61.5,27.8 .{ }^{19} \mathrm{~F} \mathrm{NMR}\left(\mathrm{CDCl}_{3}\right) \delta$-62.7. HRMS for $\mathrm{C}_{20} \mathrm{H}_{26} \mathrm{~F}_{3} \mathrm{NO}_{4} \mathrm{M}$ $+\mathrm{H}=402.18922$; found $(\mathrm{Cl}+)$ 402.18911.

(Table 4, entry 4). The general procedure was followed with $p$-bromo cinnamyl methyl carbonate $(135 \mathrm{mg}, 0.499 \mathrm{mmol})$ and $\mathrm{LiN}(\mathrm{Boc})_{2}(134.2 \mathrm{mg}, 0.601 \mathrm{mmol})$. The reaction 
was conducted at room temperature for $10 \mathrm{~h} .{ }^{1} \mathrm{H}$ NMR analysis of the crude reaction mixture indicated the ratio of regioisomers (b/l) to be 90/10. The mixture was purified by flash column chromatography on silica gel ( $9 \%$ ethyl acetate in

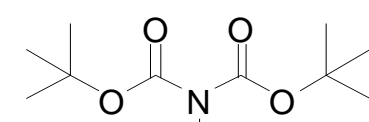
hexanes) to give the title compound (154.4 mg, 75\%). HPLC analysis after monodeprotection via treatment with trifluoroacetic $\mathrm{Br}$ acid indicated that the enantiomeric excess of the product was $92 \%$ [Diacel CHIRALCEL OD-H $(0.46 \mathrm{~cm} \times 25 \mathrm{~cm})$ hexanes/2propanol $=99 / 1 ;$ flow rate $=1.0 \mathrm{~mL} / \mathrm{min}$; detection wavelength $=210 \mathrm{~nm}] .{ }^{1} \mathrm{H}$ NMR $\left(\mathrm{CDCl}_{3}\right) \delta 8.51$ (d, $\left.J=8.5 \mathrm{~Hz}, 2 \mathrm{H}\right), 7.19(\mathrm{~d}, J=8.7 \mathrm{~Hz}, 2 \mathrm{H}$ ), 6.32 (ddd, $J=7.9,10.2$, $17.3 \mathrm{~Hz}, 1 \mathrm{H}$ ), $5.83(\mathrm{~d}, J=7.9 \mathrm{~Hz}, 1 \mathrm{H}), 5.36(\mathrm{~d}, J=10.2 \mathrm{~Hz}, 1 \mathrm{H}), 5.32(\mathrm{~d}, J=17.2 \mathrm{~Hz}$, 1H), $1.38(\mathrm{~s}, 18 \mathrm{H}) .{ }^{13} \mathrm{C} \mathrm{NMR}\left(\mathrm{CDCl}_{3}\right) \delta 152.3,139.4,134.9,131.2,128.3,120.8,120.0$, 82.6, 61.3, 27.8. Anal. Calcd. for $\left[\mathrm{C}_{19} \mathrm{H}_{26} \mathrm{BrNO}_{4}\right]$ : C, 55.35; $\mathrm{H}, 6.36 ; \mathrm{N}, 3.40$ Found: $\mathrm{C}$, $55.72 ; \mathrm{H}, 6.50 ; \mathrm{N}, 3.36$.

(Table 4, entry 5). The general procedure was followed with $(E)$-hex-2-enyl methyl carbonate $(79.7 \mathrm{mg}, 0.504 \mathrm{mmol})$ and $\operatorname{LiN}(\mathrm{Boc})_{2}(133.3 \mathrm{mg}, 0.597 \mathrm{mmol})$ with $4 \mathrm{~mol} \%$ iridium and ligand instead of $2 \mathrm{~mol} \%$. The reaction was conducted $\mathrm{C}_{\mathrm{O}}^{\mathrm{O}} \mathrm{II}_{\mathrm{O}} K$ at room temperature for $24 \mathrm{~h}$. ${ }^{1} \mathrm{H}$ NMR analysis of the crude reaction mixture indicated the ratio of regioisomers (b/l) to be 93/3. The mixture was purified by flash column chromatography on silica gel ( $7 \%$ ethyl acetate in hexanes) to give the title compound (117.8 mg, $78 \%)$. HPLC analysis after monodeprotection via treatment with trifluoroacetic acid indicated that the enantiomeric excess of the product was $96 \%$ [Diacel CHIRALCEL AS-H $(0.46 \mathrm{~cm} \times 25$ $\mathrm{cm}$ ) hexanes $/ 2$-propanol $=99.25 / 0.75$; flow rate $=1.0 \mathrm{~mL} / \mathrm{min}$; detection wavelength $=$

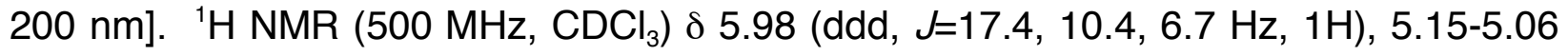
$(\mathrm{m}, 2 \mathrm{H}), 4.61(\mathrm{~m}, 1 \mathrm{H}), 1.86-1.78(\mathrm{~m}, 1 \mathrm{H}), 1.68-1.59(\mathrm{~m}, 1 \mathrm{H}), 1.47(\mathrm{~s}, 18 \mathrm{H}), 1.34-1.26$ (m, 2H), 0.90 (t, $J=7.3 \mathrm{~Hz}, 3 \mathrm{H}) .{ }^{13} \mathrm{C} \mathrm{NMR}\left(\mathrm{CDCl}_{3}\right) \delta$ 153.0, 137.9, 116.0, 81.9, 58.9, 34.6, 28.0, 19.5, 13.7. Anal. Calcd. for $\left[\mathrm{C}_{16} \mathrm{H}_{29} \mathrm{NO}_{4}\right]$ : C, 59.26; $\mathrm{H}, 4.97 ; \mathrm{N}, 5.76$ Found: C, $59.21 ; \mathrm{H}, 4.87 ; \mathrm{N}, 5.74$. 
(Table 4, entry 6). The general procedure was followed with (E)-4-methylpent-2-enyl methyl carbonate $(79.2 \mathrm{mg}, 0.501 \mathrm{mmol})$ and $\operatorname{LiN}(B o c){ }_{2}(134.3 \mathrm{mg}, 0.602 \mathrm{mmol})$. The

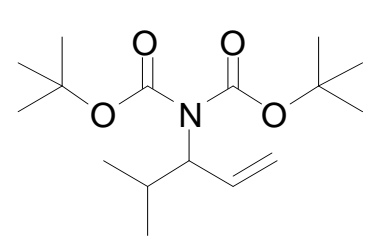
reaction was conducted at room temperature for $12 \mathrm{~h} .{ }^{1} \mathrm{H}$ NMR analysis of the crude reaction mixture indicated the ratio of regioisomers $(b / l)$ to be $89 / 11$. The mixture was purified by flash column chromatography on silica gel ( $8 \%$ ethyl acetate in hexanes)

to give the title compound (63.1 mg, 42\%). HPLC analysis after monodeprotection via treatment with trifluoroacetic acid indicated that the enantiomeric excess of the product was $97 \%$ [Diacel CHIRALCEL OJ-H $(0.46 \mathrm{~cm} \times 25 \mathrm{~cm})$ hexanes/2-propanol = 99.75/0.25; flow rate $=1.0 \mathrm{~mL} / \mathrm{min}$; detection wavelength $=200 \mathrm{~nm}] .{ }^{1} \mathrm{H} \mathrm{NMR}\left(\mathrm{CDCl}_{3}\right) \delta$ 6.03 (ddd, $J=8.1,10.2,17.3 \mathrm{~Hz}, 1 \mathrm{H}$ ), 5.17 (d, $J=17.3 \mathrm{~Hz}, 1 \mathrm{H}$ ), $5.12(\mathrm{~d}, J=10.2,1 \mathrm{H}$ ), 4.21-4.07 (m, 1H), 2.28-2.12 (m, 1H), $1.47(\mathrm{~s}, 18 \mathrm{H}), 0.88(\mathrm{~d}, J=6.7,11.9 \mathrm{~Hz}, 6 \mathrm{H}) .{ }^{13} \mathrm{C}$ NMR $\left(\mathrm{CDCl}_{3}\right) \delta 153.3,136.9,118.0,81.9,66.6,29.8,28.0,20.7,19.3$. Anal. Calcd. for $\left[\mathrm{C}_{16} \mathrm{H}_{29} \mathrm{NO}_{4}\right]: \mathrm{C}, 64.18 ; \mathrm{H}, 9.76 ; \mathrm{N}, 4.68$ Found: $\mathrm{C}, 64.34 ; \mathrm{H}, 9.85 ; \mathrm{N}, 4.57$.

(Table 4, entry 7). The general procedure was followed with (E)-3-(furan-2-yl)allyl

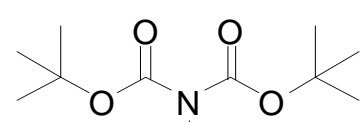
methyl carbonate $(91.7 \mathrm{mg}, 0.503 \mathrm{mmol})$ and $\mathrm{LiN}(\mathrm{Boc})_{2}(134 \mathrm{mg}$, $0.599 \mathrm{mmol}$ ) and using L1 instead of L5. The reaction was conducted at room temperature for $5 \mathrm{~h}$. ${ }^{1} \mathrm{H}$ NMR analysis of the crude reaction mixture indicated the ratio of regioisomers $(b / l)$ to be $77 / 23$. The mixture was purified by flash column chromatography on silica gel $(6 \%$ ethyl acetate in hexanes) to give the title compound (96.7 mg, 59\%). HPLC analysis after monodeprotection via treatment with trifluoroacetic acid indicated that the enantiomeric excess of the product was 92\% [Diacel CHIRALCEL OJ-H $(0.46 \mathrm{~cm} \times 25 \mathrm{~cm})$ hexanes $/ 2$-propanol $=99.25 / 0.75 ;$ flow rate $=1.0 \mathrm{~mL} / \mathrm{min}$; detection wavelength $=220$ nm]. ${ }^{1} \mathrm{H}$ NMR $\left(500 \mathrm{MHz}, \mathrm{CDCl}_{3}\right) \delta 7.33(\mathrm{~m}, 1 \mathrm{H}), 6.33-6.29(\mathrm{~m}, 1 \mathrm{H}), 6.28(\mathrm{dd}, J=10.1$, $7.2 \mathrm{~Hz}, 1 \mathrm{H}), 6.16-6.15(\mathrm{~m}, 1 \mathrm{H}), 5.93(\mathrm{~d}, J=7.2 \mathrm{~Hz}, 1 \mathrm{H}), 5.35(\operatorname{app} d, J=17.2 \mathrm{~Hz}, 1 \mathrm{H})$, 5.31 (app d, $J=10.2 \mathrm{~Hz}, 1 \mathrm{H}), 1.42(\mathrm{~s}, 18 \mathrm{H}) .{ }^{13} \mathrm{C} \mathrm{NMR}\left(\mathrm{CDCl}_{3}\right) \delta$ 152.8, 152.1, 141.3, 133.8, 118.9, 110.3, 106.7, 82.5, 56.4, 27.8. Anal. Calcd. for $\left[\mathrm{C}_{17} \mathrm{H}_{25} \mathrm{NO}_{5}\right]: \mathrm{C}, 59.26 ; \mathrm{H}$, 4.97 ; N, 5.76 Found: C, 59.21; H, 4.87; N, 5.74. 


\section{References}

(1) Crabtree, R. H.; Quirk, J. M.; Felkin, H.; Fillebeenkhan, T. Synthesis and Reactivity in Inorganic and Metal-Organic Chemistry 1982, $12,407$.

(2) (a) Alexakis, A.; Rosset, S.; Allamand, J.; March, S.; Guillen, F.; Benhaim, C. Synlett 2001, 1375; (b) Polet, D.; Alexakis, A.; Tissot-Croset, K.; Corminboeuf, C.; Ditrich, K. Chem. Eur. J. 2006, 12, 3596; (c) Leitner, A.; Shekhar, S.; Pouy, M. J.; Hartwig, J. F. J. Am. Chem. Soc. 2005, 127, 15506.

(3) Blanchette, M. A.; Choy, W.; Davis, J. T.; Essenfeld, A. P.; Masamune, S.; Roush, W. R.; Sakai, T. Tetrahedron Lett. 1984, 25, 2183.

(4) Weix, D. J.; Hartwig, J. F. J. Am. Chem. Soc. 2007, 129, 7720. 
$\int_{\text {bis(1-phenylallyl)amine }}^{\mathrm{N}} \mathrm{C}^{\mathrm{Ph}}$
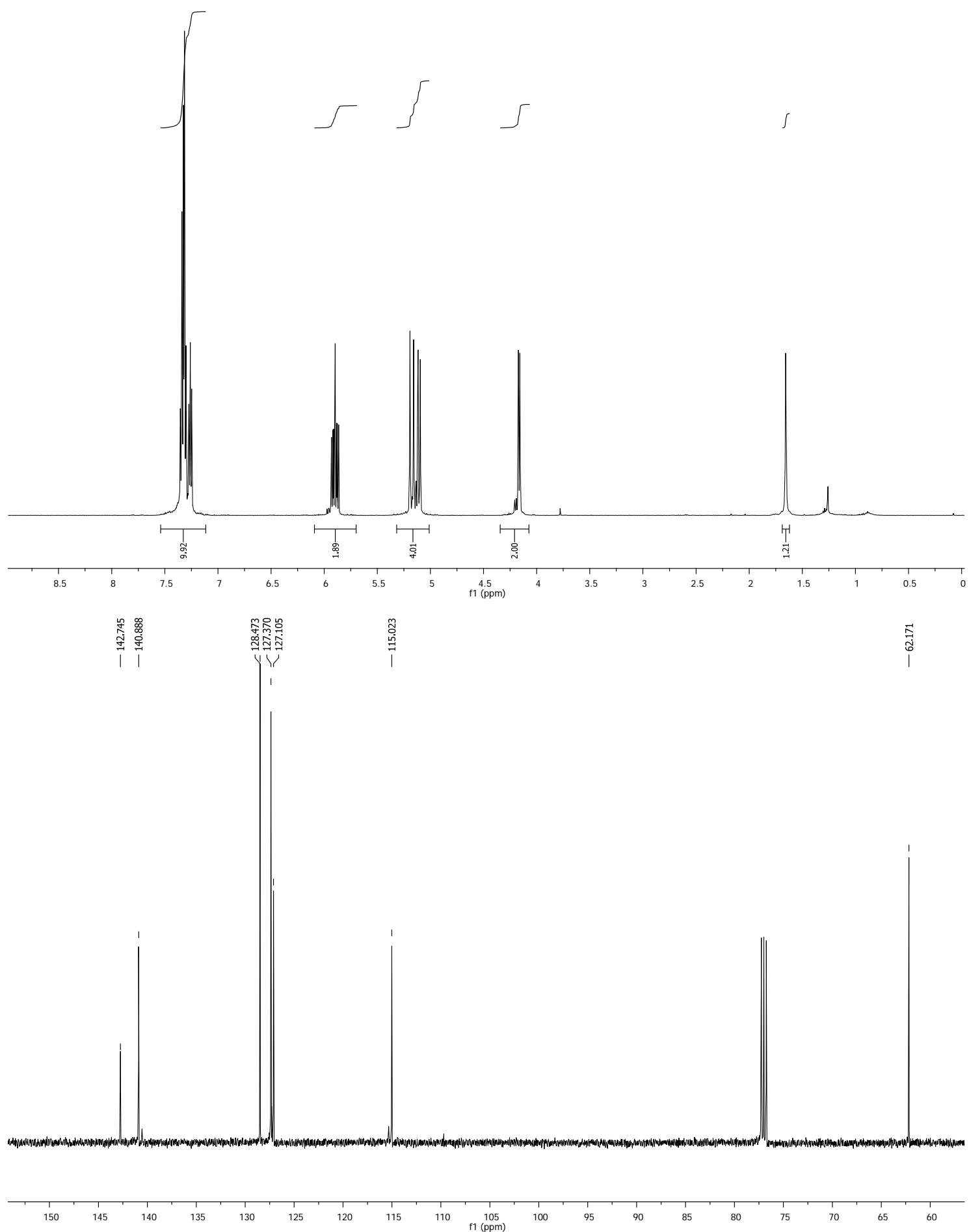
<smiles>C=CC(NC(=O)C(F)(F)F)c1ccccc1</smiles>
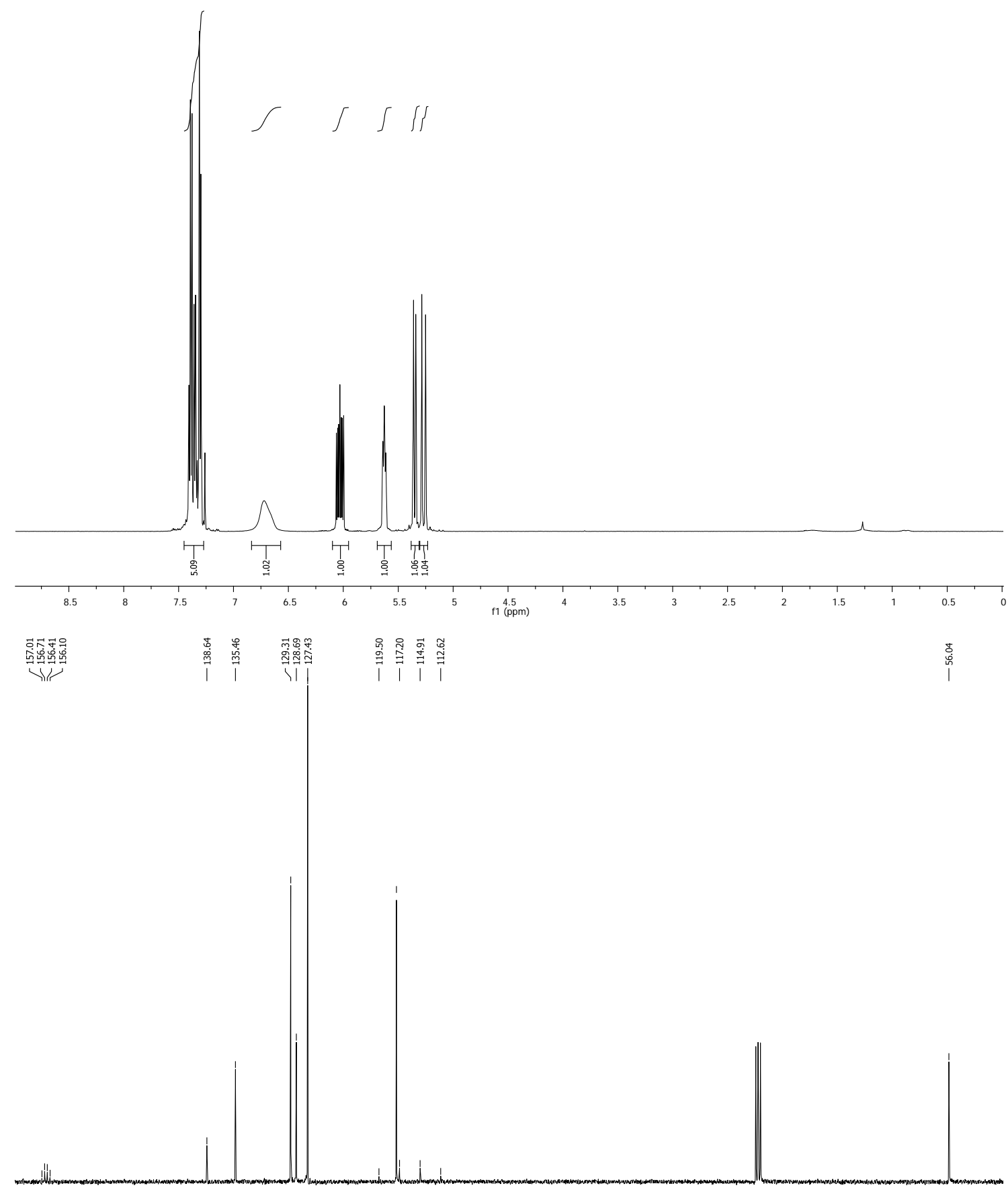

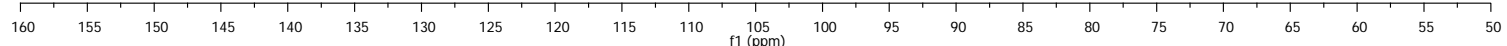


Table 2, entry 2
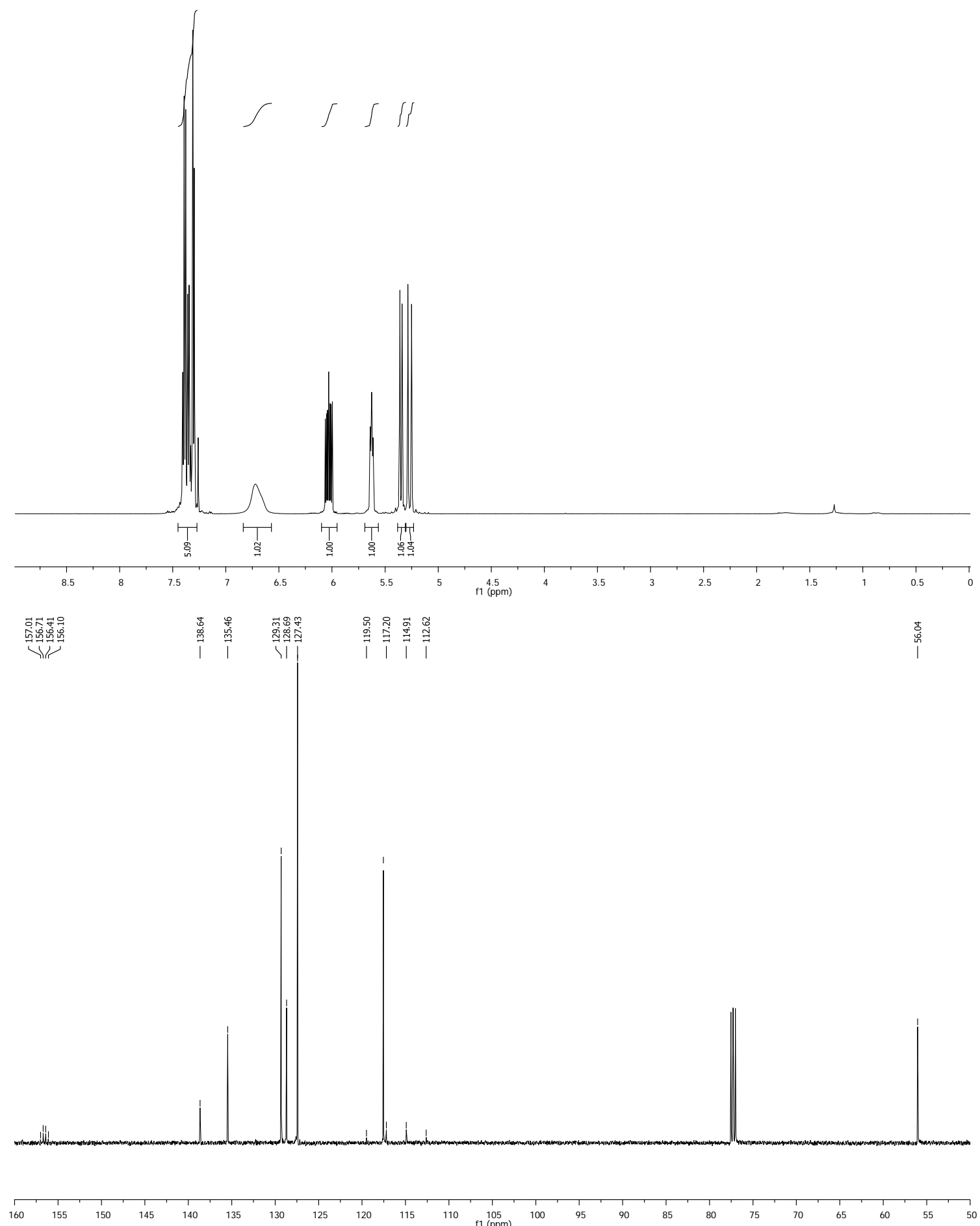
<smiles>C=CC(NC(=O)C(F)(F)F)c1ccc(OC)cc1</smiles>

Table 2, entry 3

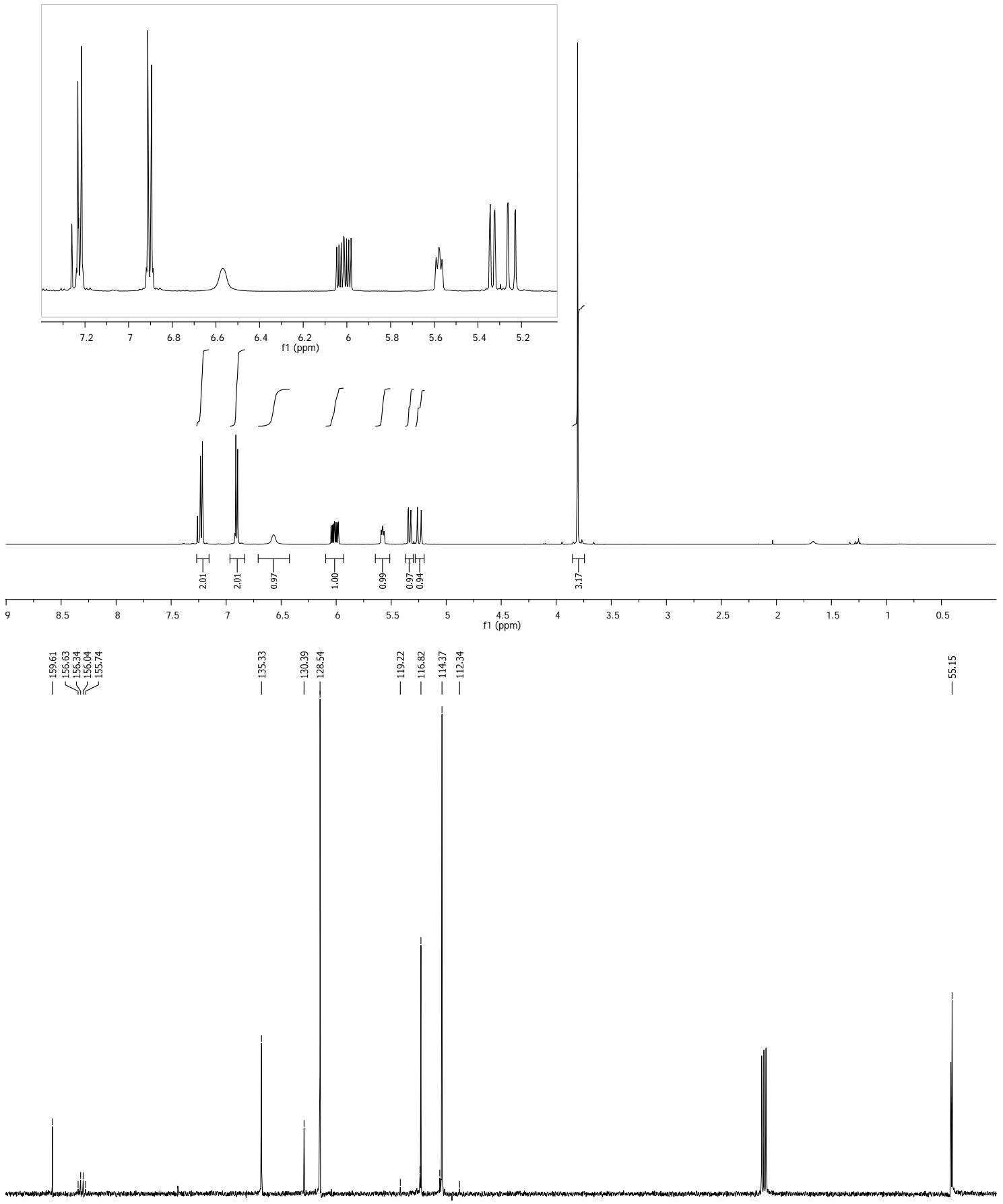

$\begin{array}{lllllllllllllllllllllllllllllllllllll}165 & 160 & 155 & 150 & 145 & 140 & 135 & 130 & 125 & 120 & 115 & 110 & 105 & 100 & 95 & 90 & 85 & 80 & 75 & 70 & 65 & 60 & 55 & 50\end{array}$ 
<smiles>C=CC(NC(=O)C(F)(F)F)c1ccc(Cl)cc1</smiles>

Table 2, entry 4

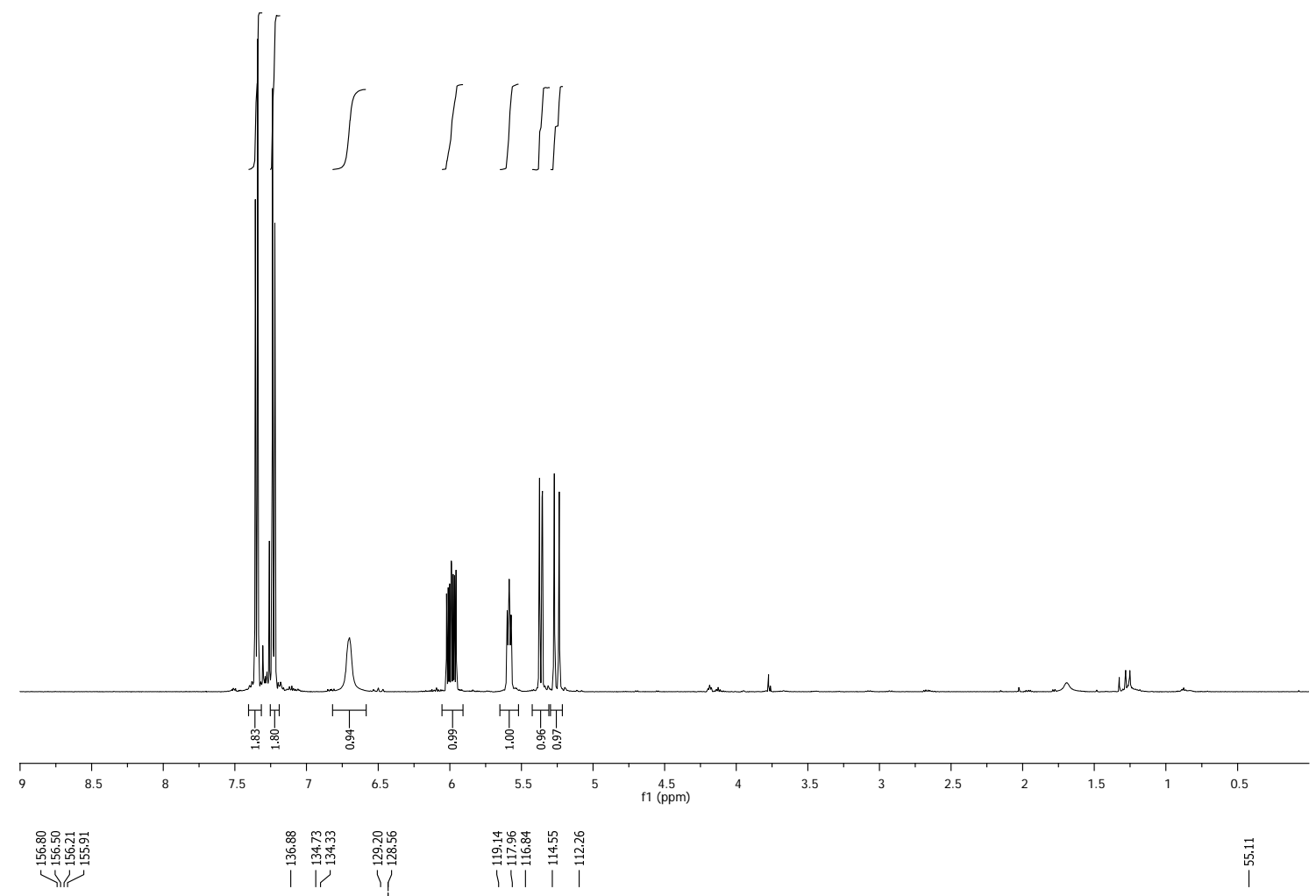


<smiles>C=CC(NC(=O)C(F)(F)F)c1ccc(Br)cc1</smiles>

Table 2, entry 5
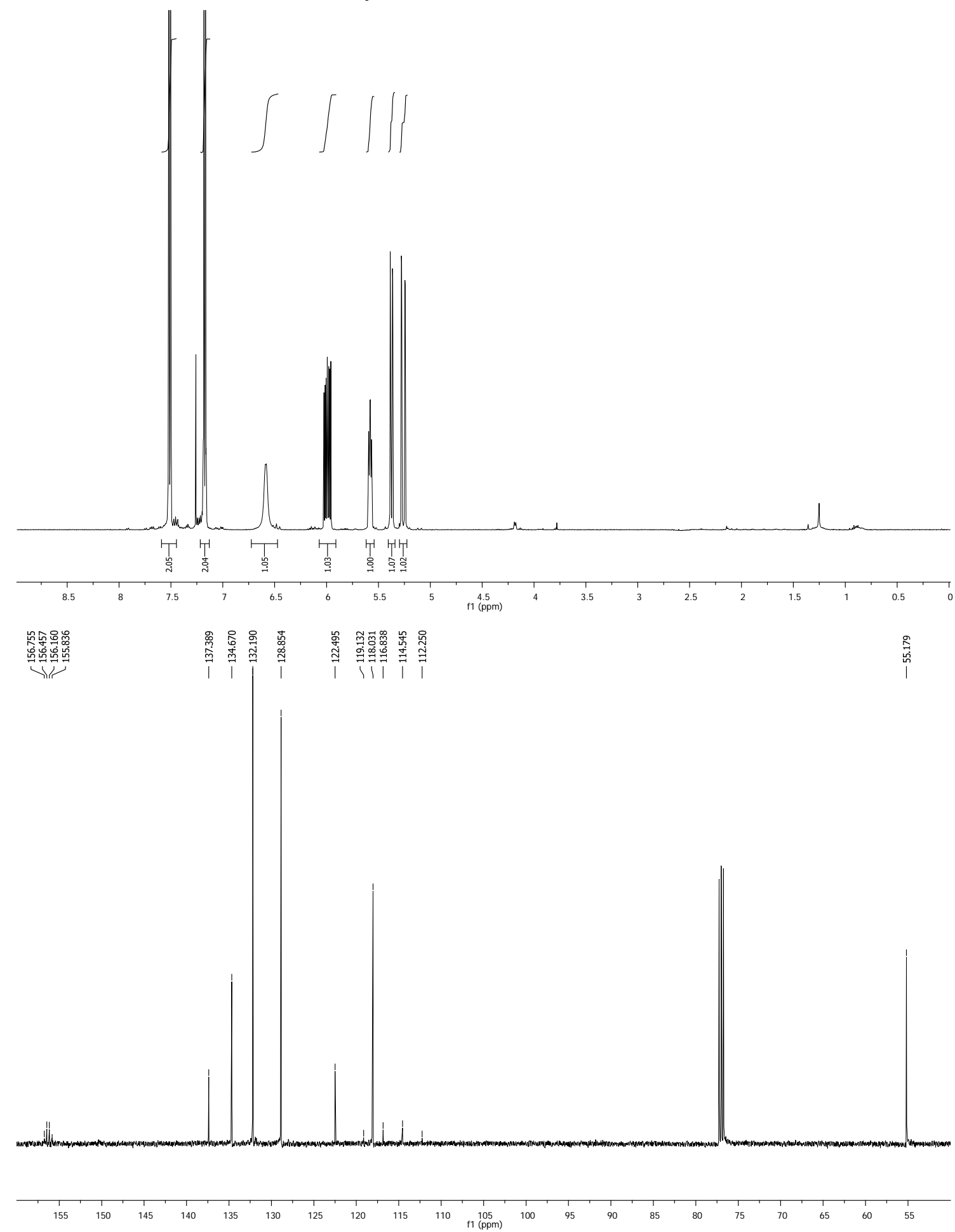
<smiles>C=CC(NC(=O)C(F)(F)F)c1ccccc1F</smiles>

Table 2, entry 6
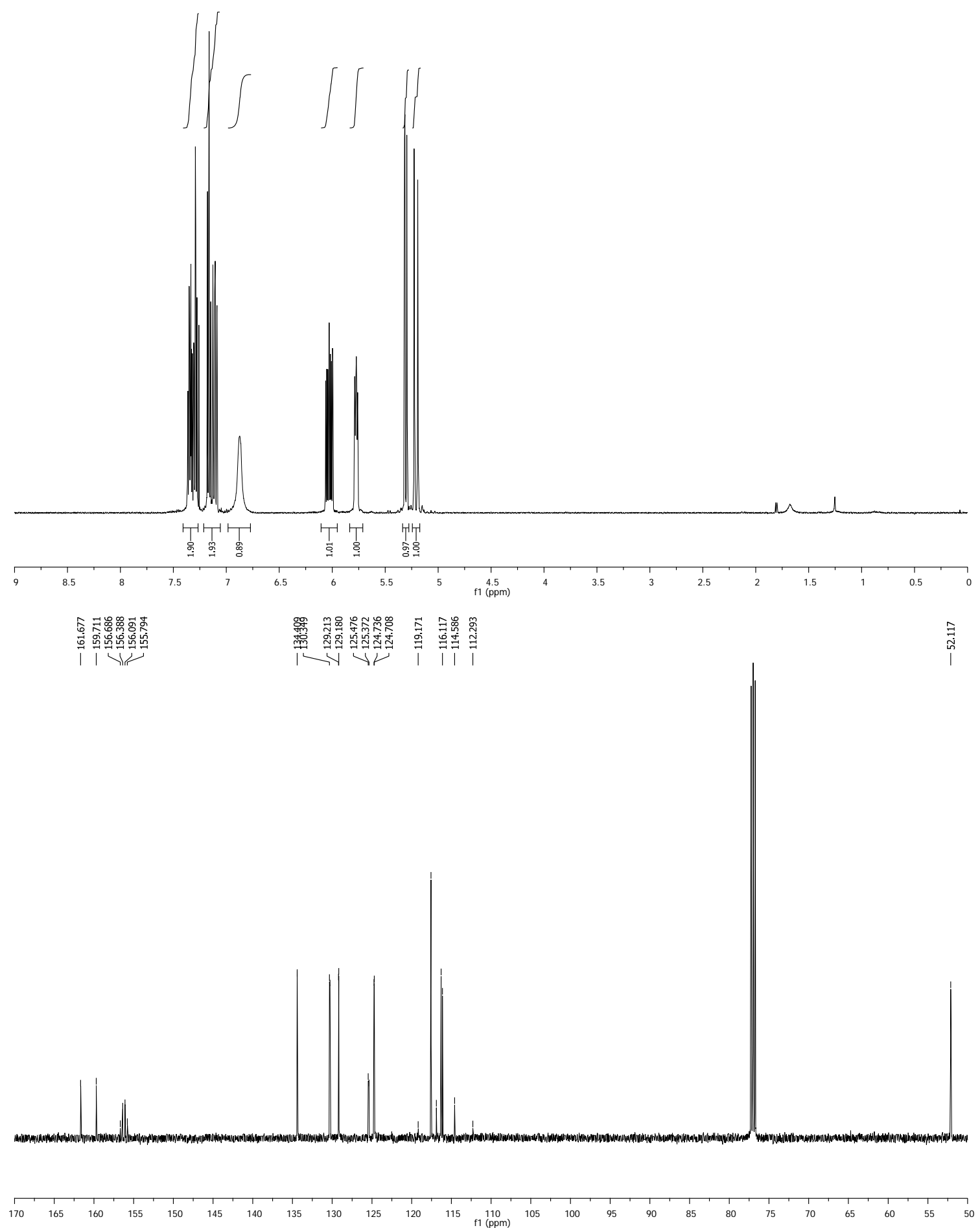


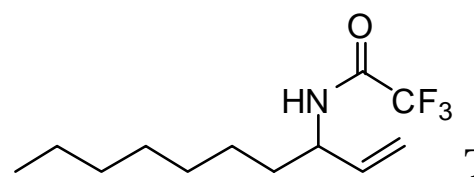

Table 2, entry 7
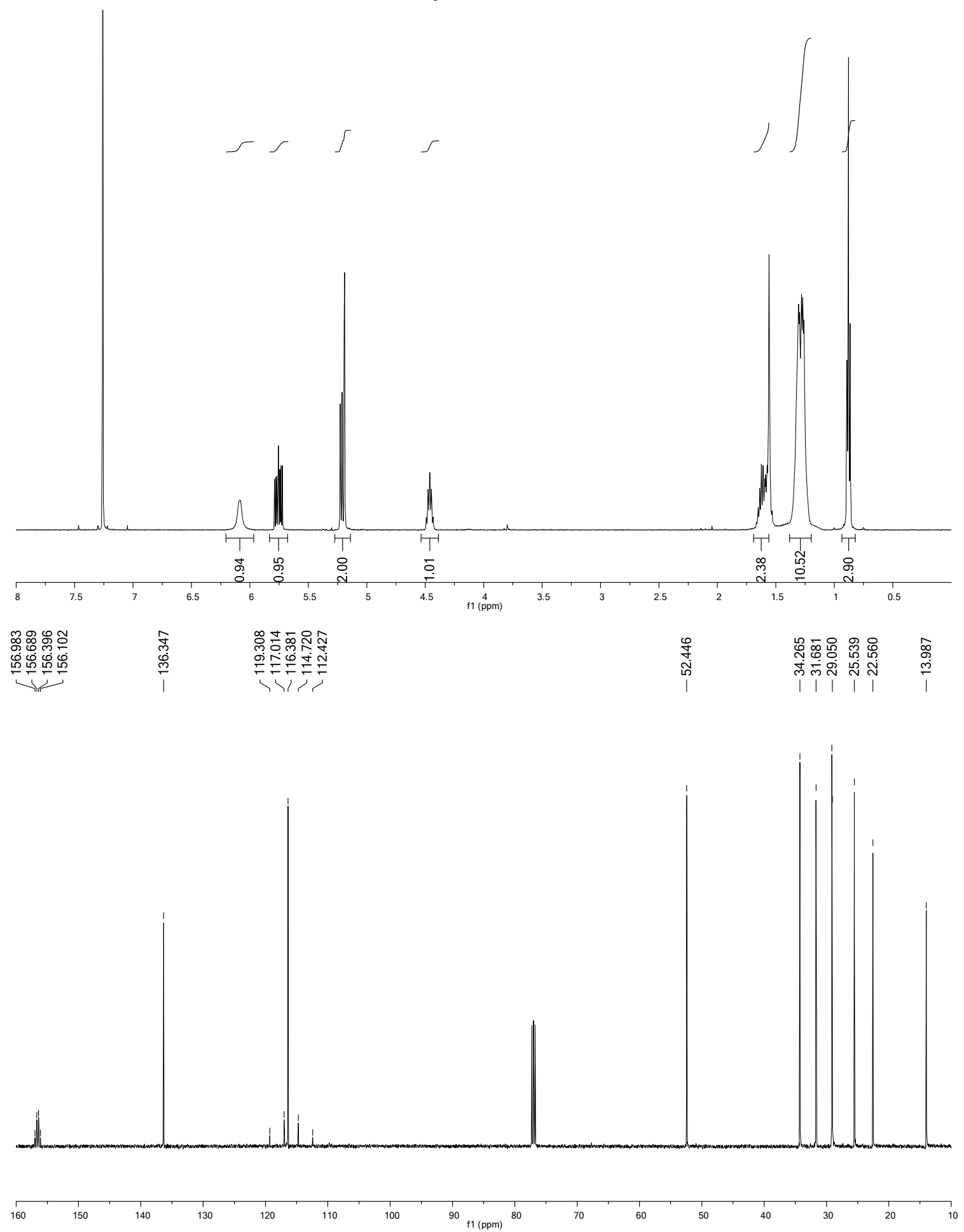
Table 2, entry 8
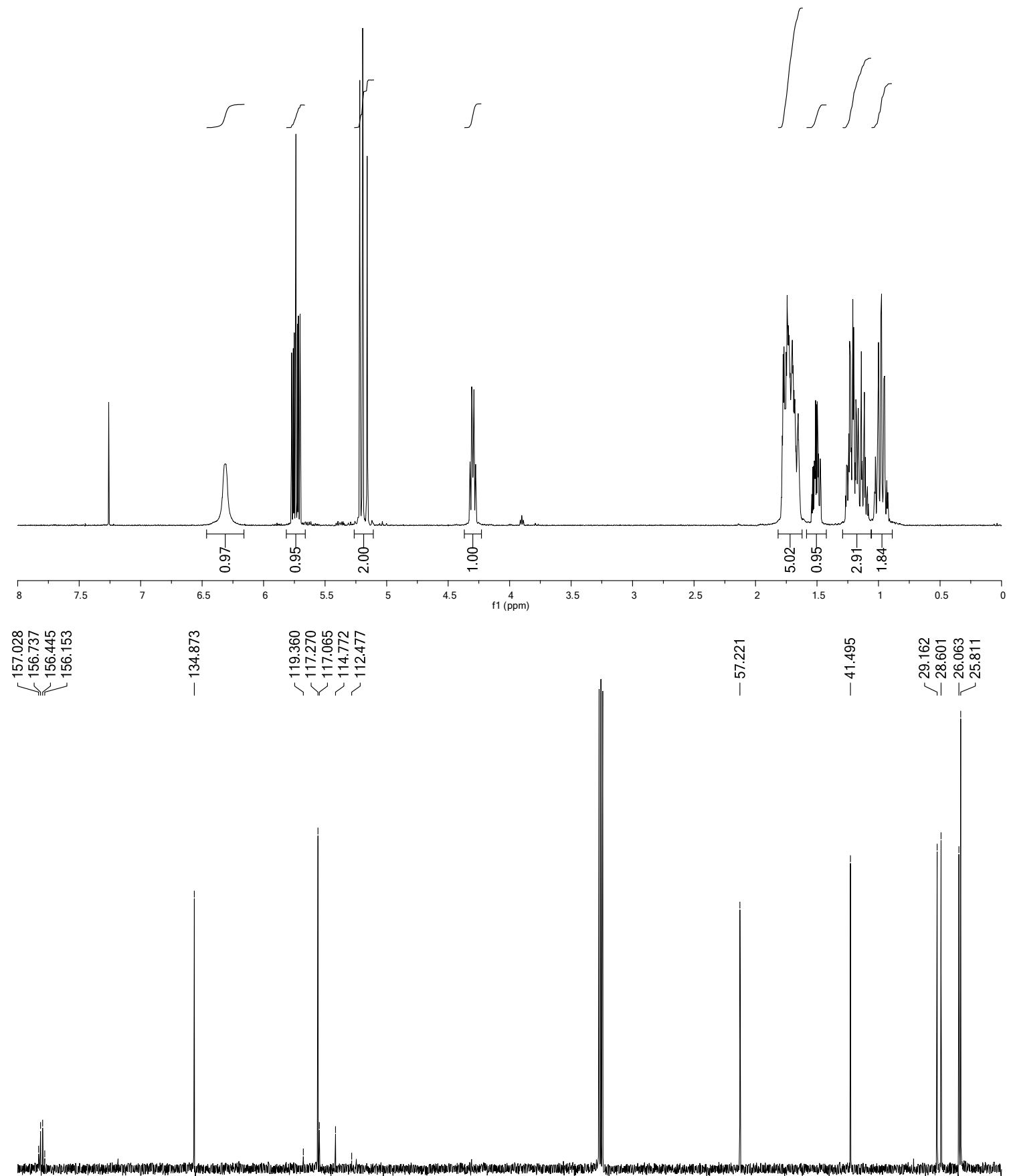

$\begin{array}{llllllllllllllllllllllllllllllllll}155 & 150 & 145 & 140 & 135 & 130 & 125 & 120 & 115 & 110 & 105 & 100 & 95 & \begin{array}{c}90 \\ \mathrm{f} 1(\mathrm{ppm})\end{array} & 85 & 80 & 75 & 70 & 65 & 60 & 55 & 50 & 45 & 40 & 35 & 30 & 25 & 20\end{array}$ 


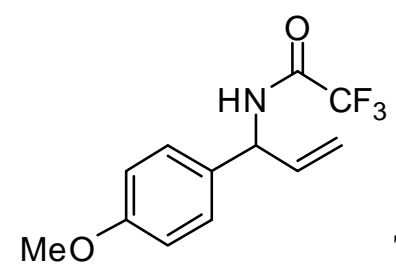

Table 4, entry 1
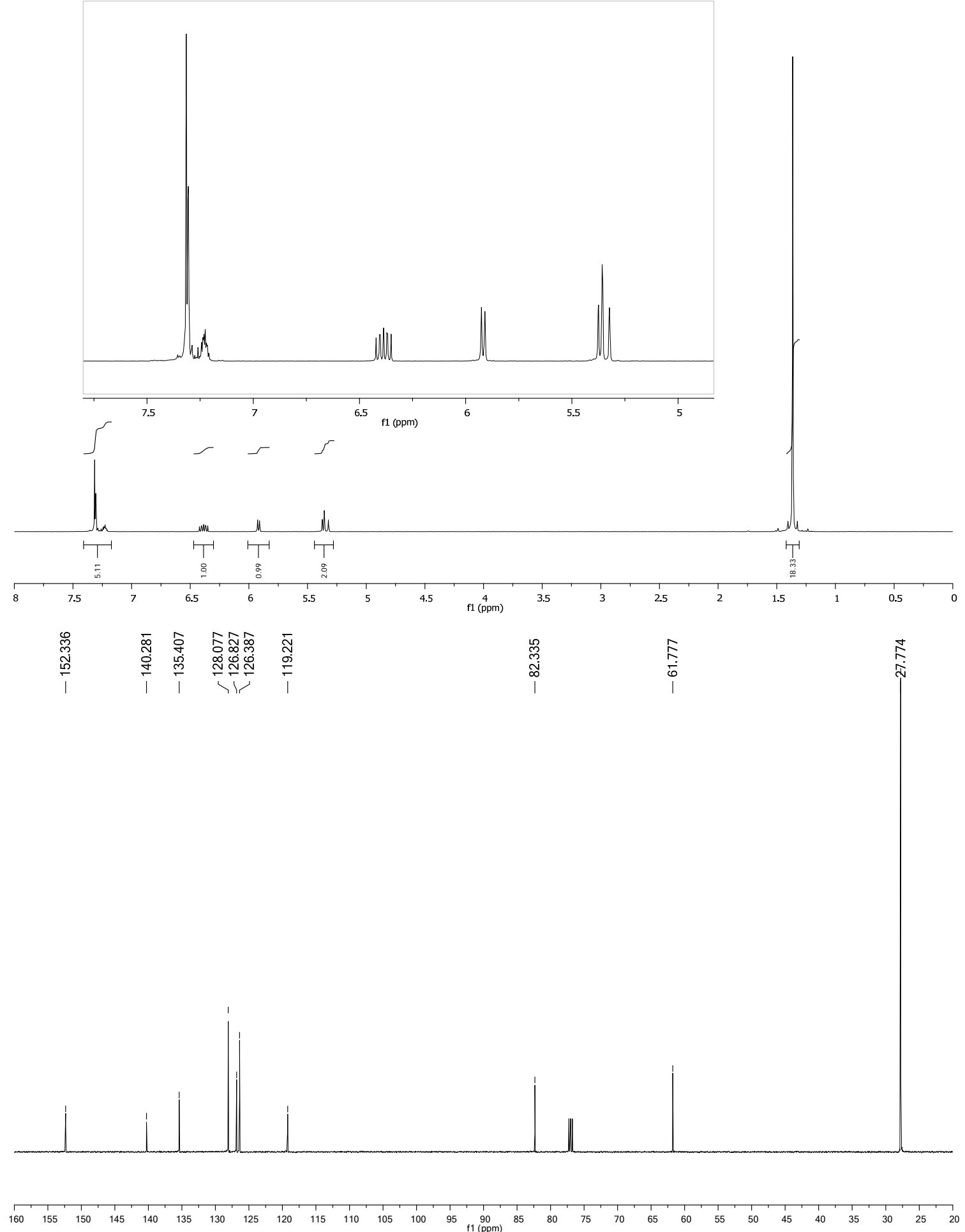


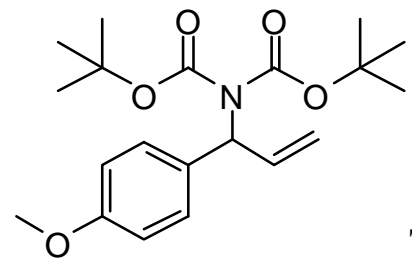

Table 4, entry 2

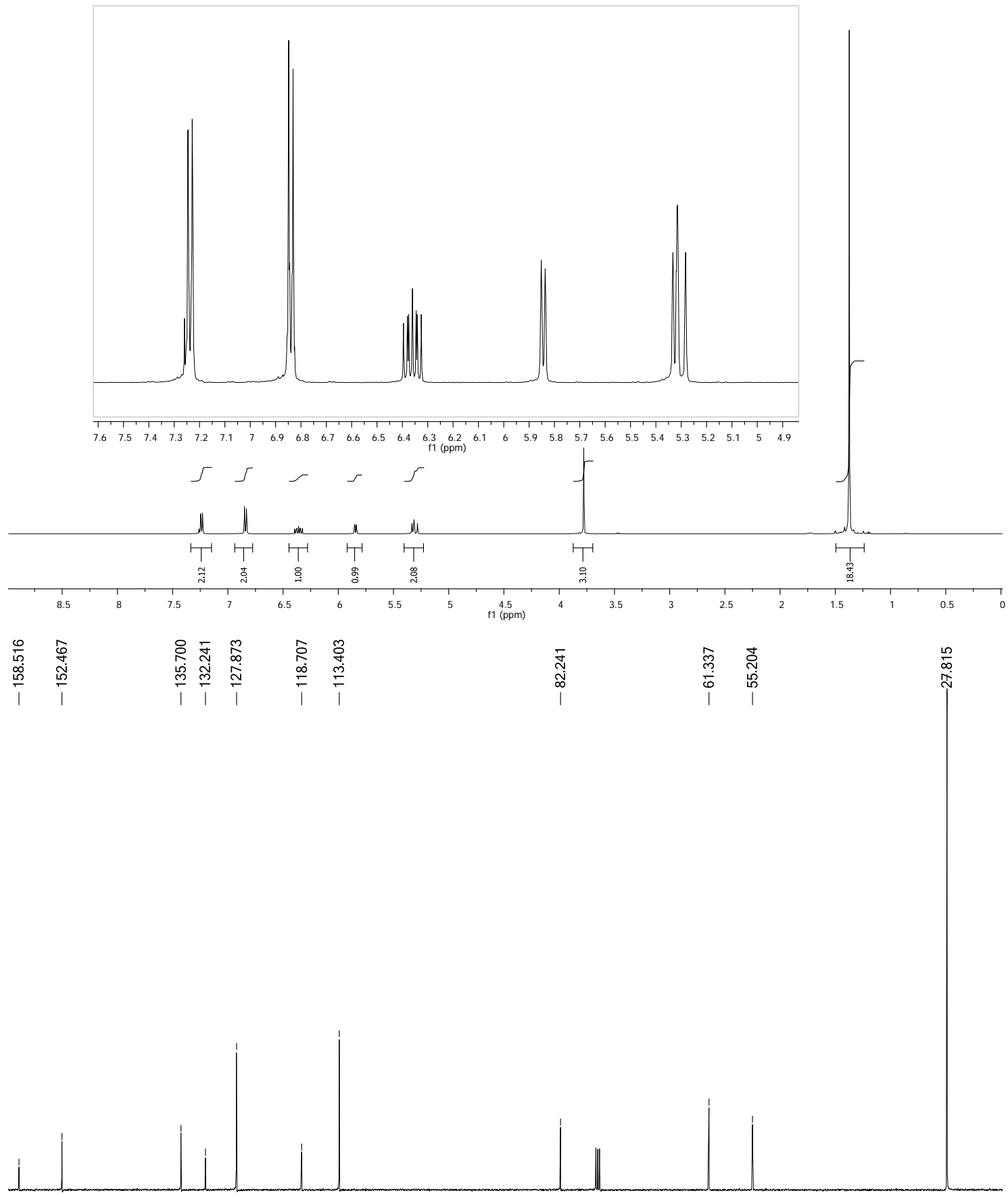

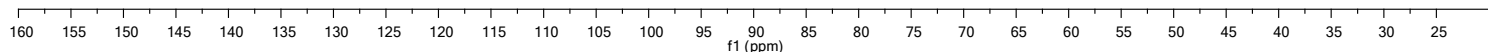




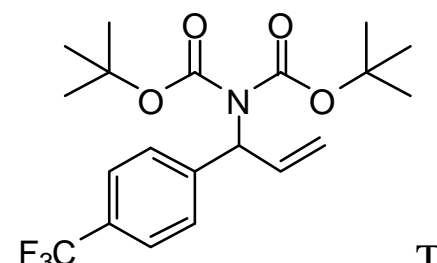

Table 4, entry 3
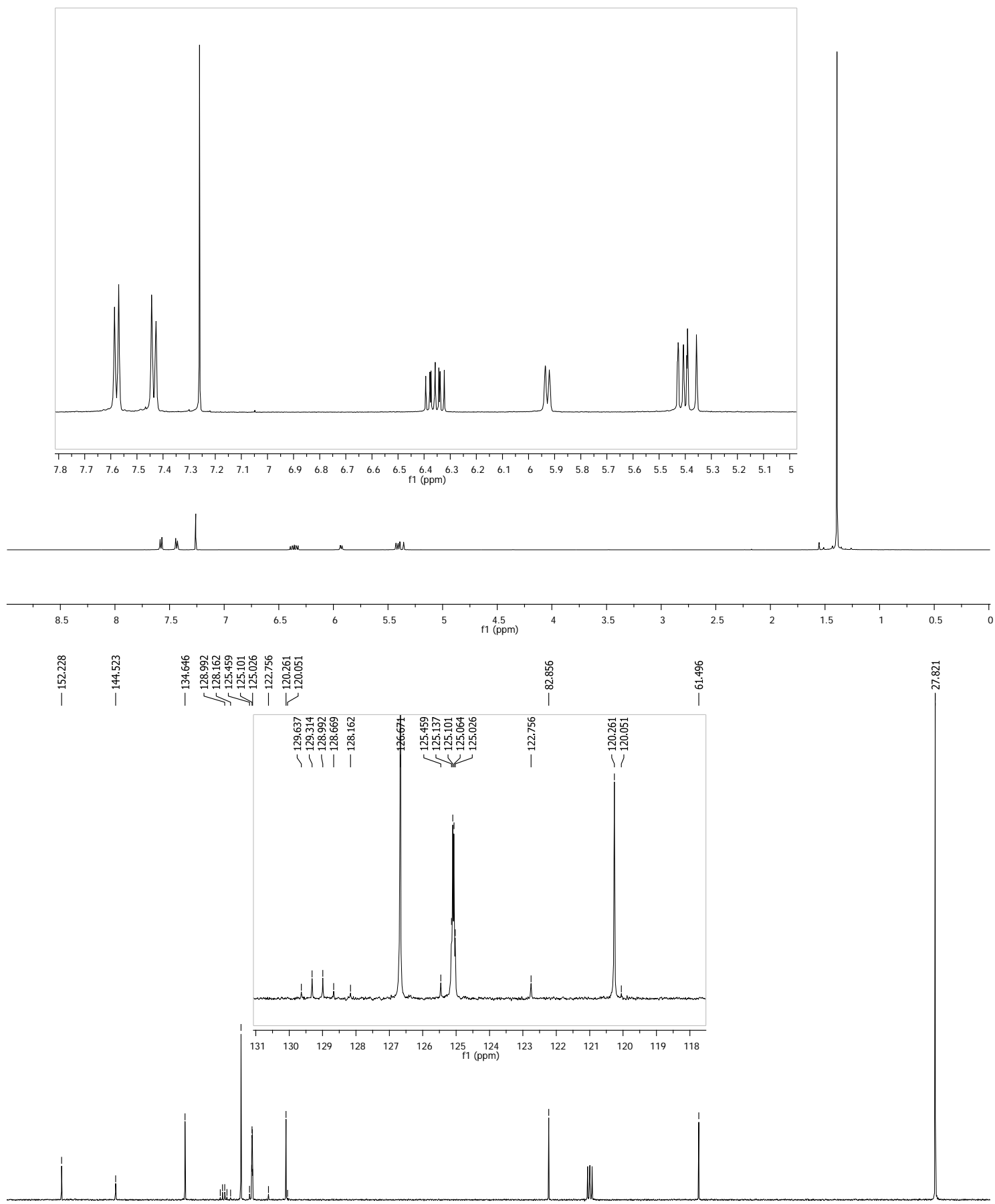

$\begin{array}{llllllllllllllllllllllllllllllllllllll}160 & 155 & 150 & 145 & 140 & 135 & 130 & 125 & 120 & 115 & 110 & 105 & 100 & 95 & 90 & 85 & 80 & 75 & 70 & 65 & 60 & 55 & 50 & 45 & 40 & 35 & 30 & 25 & \end{array}$ 

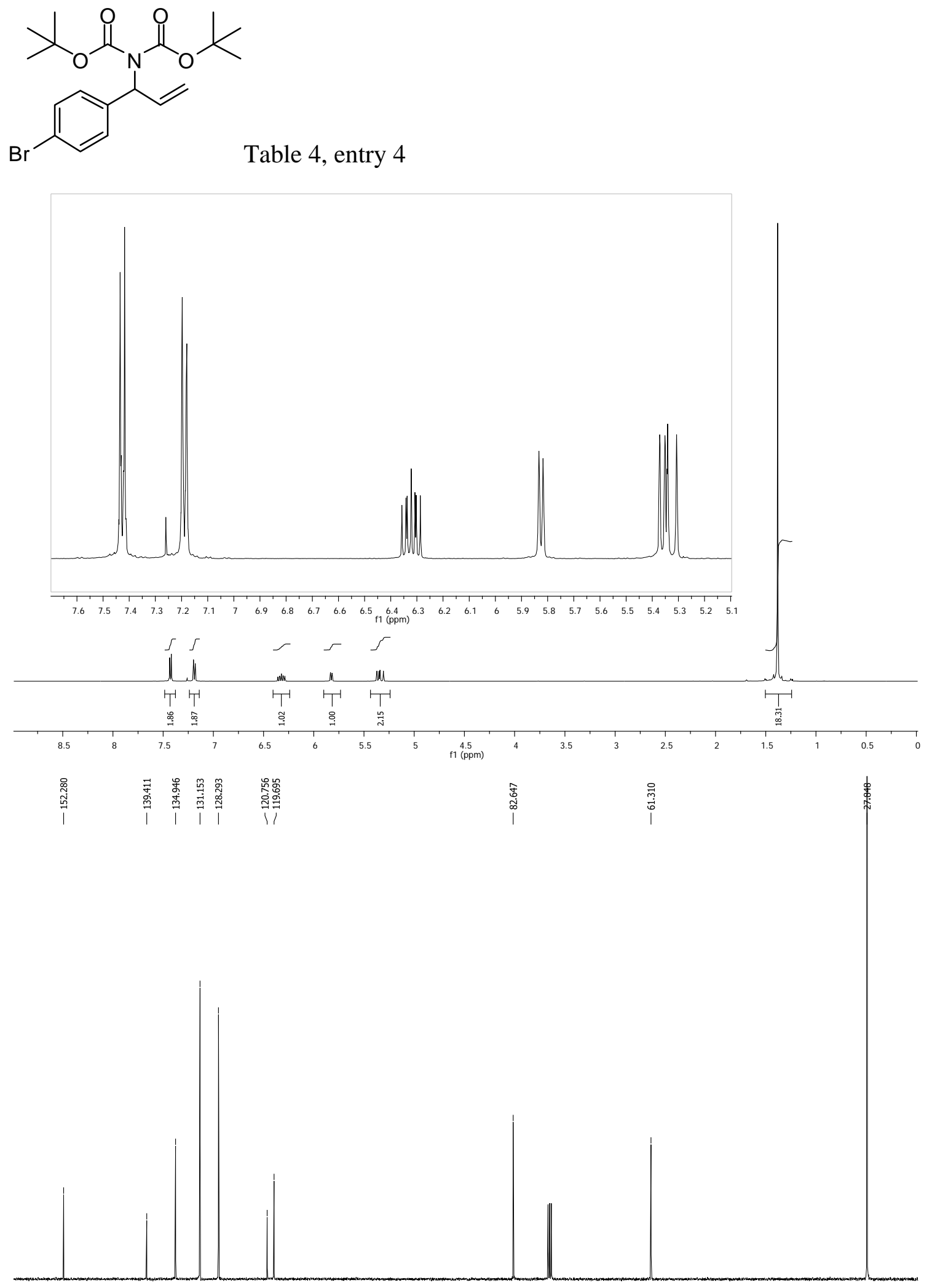

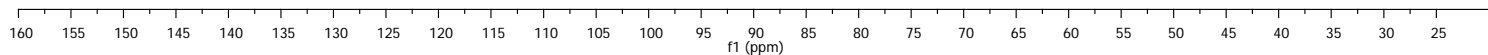




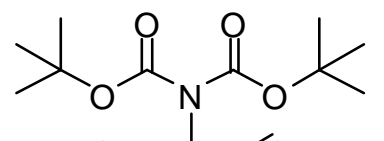

Table 4, entry 5
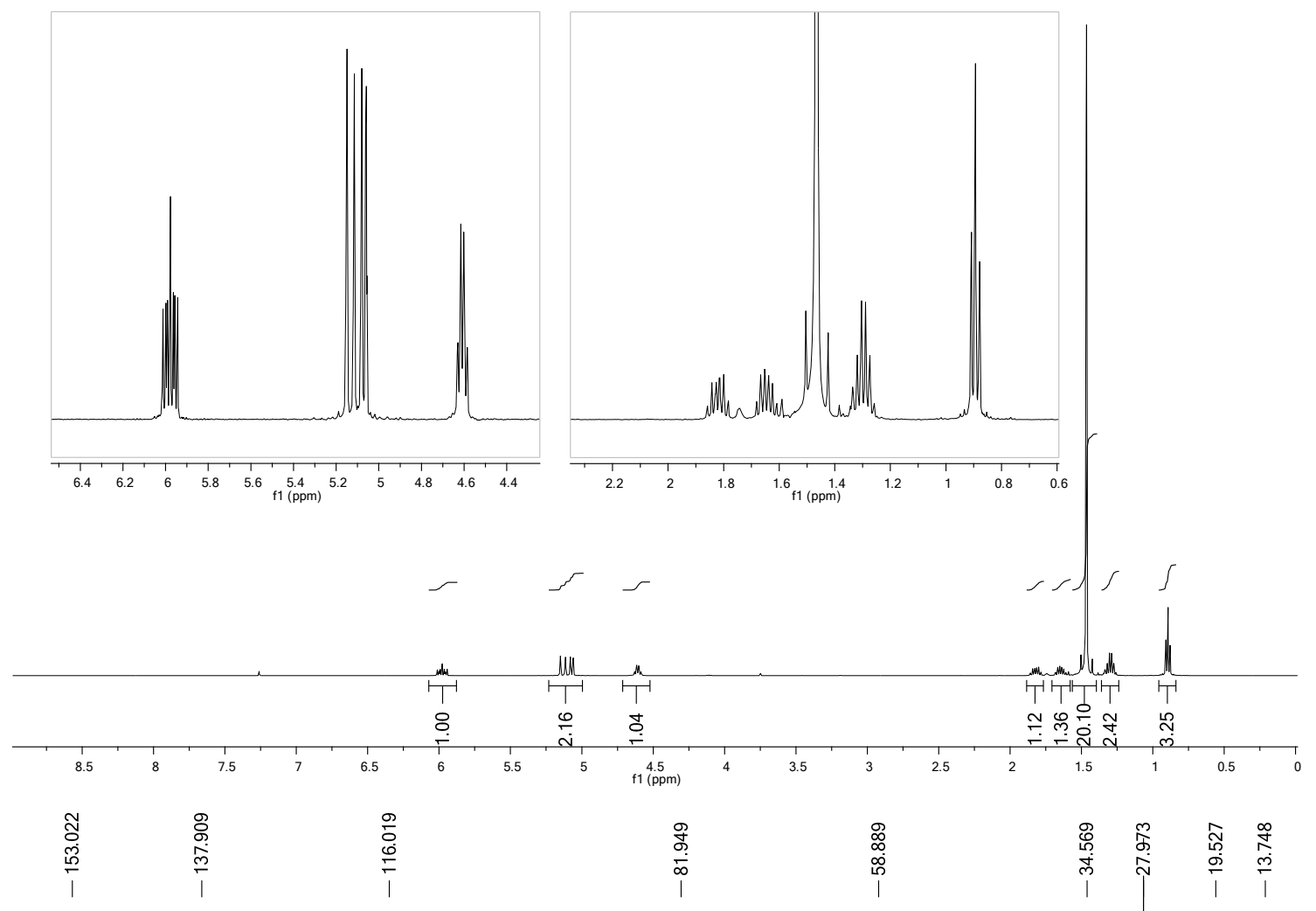

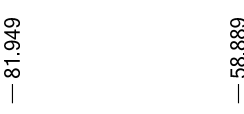
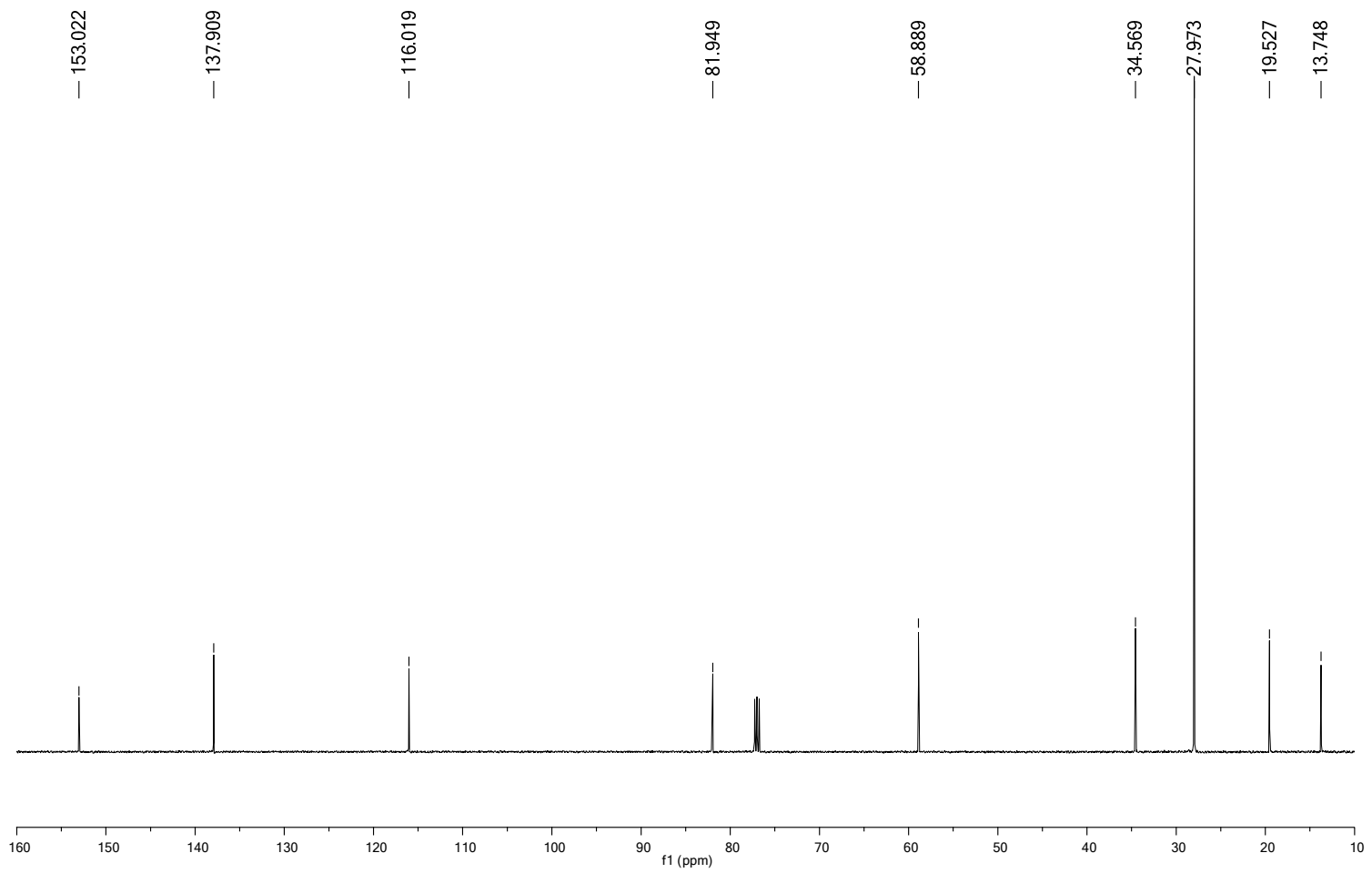
<smiles>C=CC(C(C)C)N(C(=O)OC(C)(C)C)C(=O)OC(C)(C)C</smiles>

Table 4, entry 6

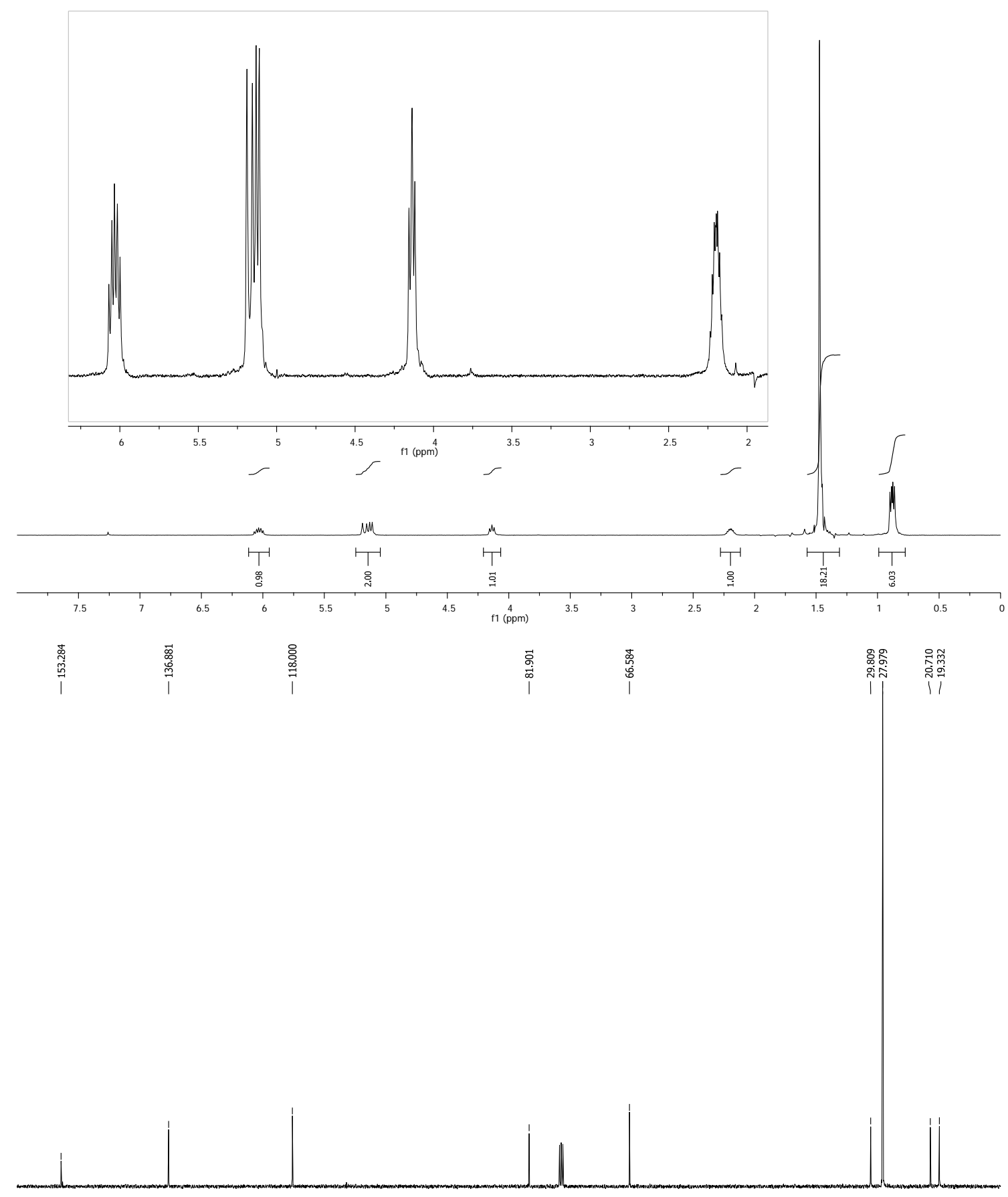

160 

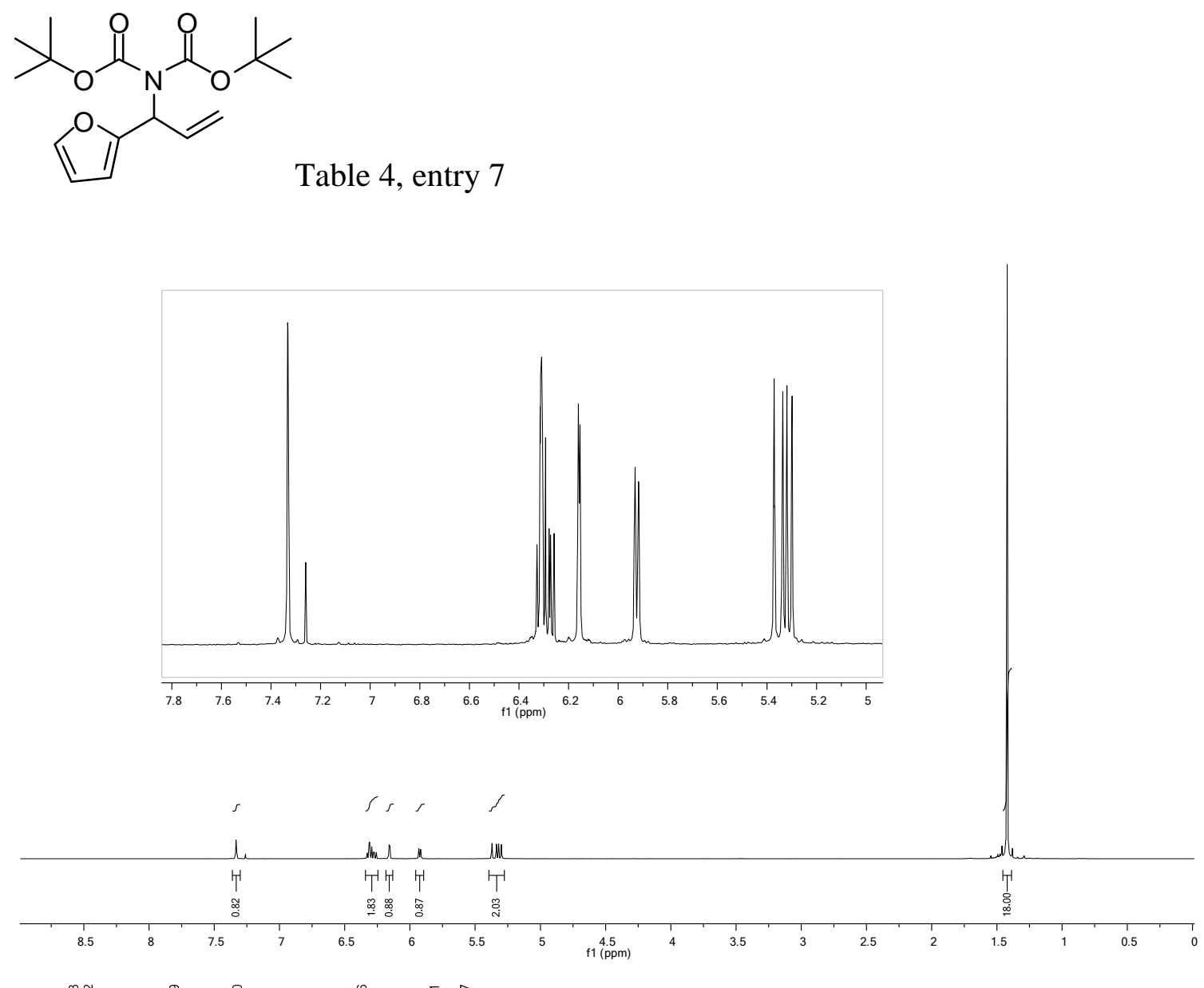

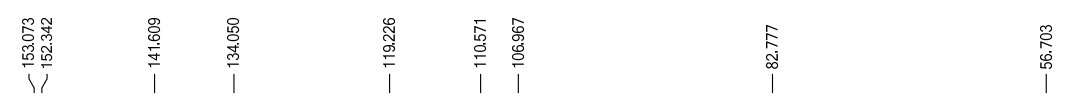
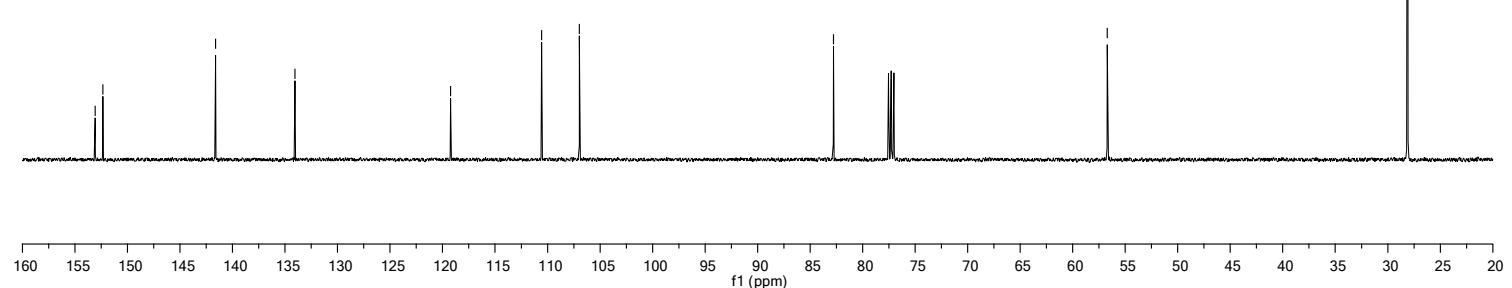